Transport coefficients and heat fluxes in non-equilibrium high-temperature flows with electronic excitation

V. A. Istomin and E. V. Kustova

Citation: Phys. Plasmas 24, 022109 (2017); doi: 10.1063/1.4975315

View online: http://dx.doi.org/10.1063/1.4975315

View Table of Contents: http://aip.scitation.org/toc/php/24/2

Published by the American Institute of Physics

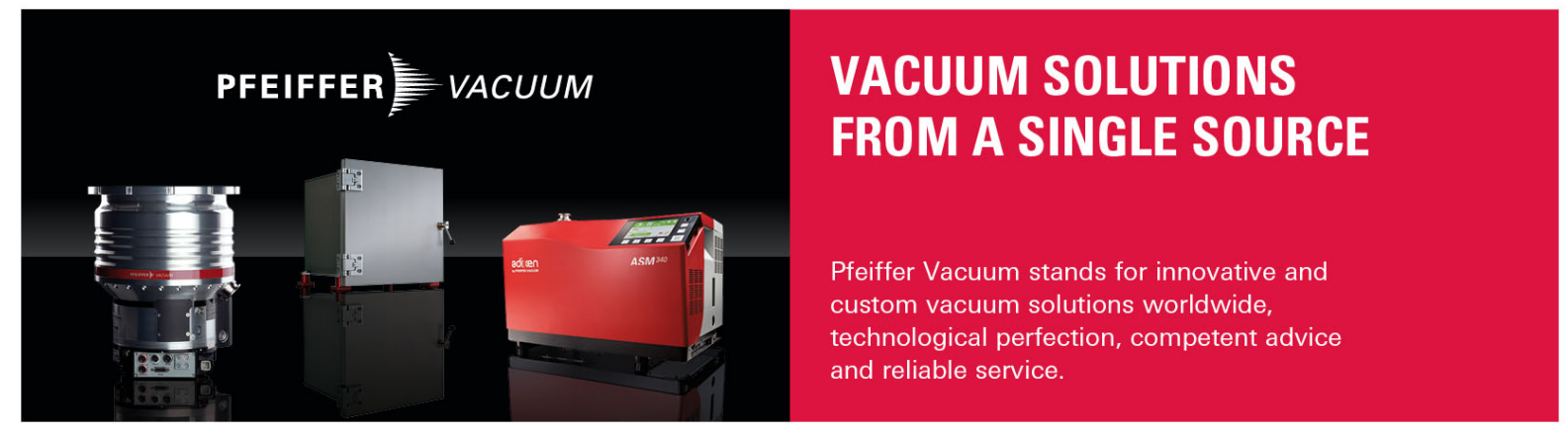




\title{
Transport coefficients and heat fluxes in non-equilibrium high-temperature flows with electronic excitation
}

\author{
V. A. Istomin and E. V. Kustova \\ Saint Petersburg State University, 7/9 Universitetskaya nab., St. Petersburg 199034, Russia
}

(Received 13 December 2016; accepted 19 January 2017; published online 7 February 2017)

The influence of electronic excitation on transport processes in non-equilibrium high-temperature ionized mixture flows is studied. Two five-component mixtures, $N_{2} / N_{2}^{+} / N / N^{+} / e^{-}$and $\mathrm{O}_{2} / \mathrm{O}_{2}^{+} / \mathrm{O} / \mathrm{O}^{+} / e^{-}$, are considered taking into account the electronic degrees of freedom for atomic species as well as the rotational-vibrational-electronic degrees of freedom for molecular species, both neutral and ionized. Using the modified Chapman-Enskog method, the transport coefficients (thermal conductivity, shear viscosity and bulk viscosity, diffusion and thermal diffusion) are calculated in the temperature range $500-50000 \mathrm{~K}$. Thermal conductivity and bulk viscosity coefficients are strongly affected by electronic states, especially for neutral atomic species. Shear viscosity, diffusion, and thermal diffusion coefficients are not sensible to electronic excitation if the size of excited states is assumed to be constant. The limits of applicability for the Stokes relation are discussed; at high temperatures, this relation is violated not only for molecular species but also for electronically excited atomic gases. Two test cases of strongly non-equilibrium flows behind plane shock waves corresponding to the spacecraft re-entry (Hermes and Fire II) are simulated numerically. Fluid-dynamic variables and heat fluxes are evaluated in gases with electronic excitation. In inviscid flows without chemical-radiative coupling, the flow-field is weakly affected by electronic states; however, in viscous flows, their influence can be more important, in particular, on the convective heat flux. The contribution of different dissipative processes to the heat transfer is evaluated as well as the effect of reaction rate coefficients. The competition of diffusion and heat conduction processes reduces the overall effect of electronic excitation on the convective heating, especially for the Fire II test case. It is shown that reliable models of chemical reaction rates are of great importance for accurate predictions of the fluid dynamic variables and heat fluxes. Published by AIP Publishing. [http://dx.doi.org/10.1063/1.4975315]

\section{INTRODUCTION}

Growing interest in theoretical studies of high-temperature reacting flows with excited electronic degrees of freedom is connected to the problems of spacecraft (re)entry to planetary atmospheres and the design of thermal protection systems, which, in particular, needs reliable data on transport processes and their influence on heat and mass transfer. During the planetary entry of an aircraft, the temperature grows sharply behind a shock wave up to thousand degrees Kelvin, which results in excitation of internal degrees of freedom, dissociation and ionization processes, and radiation. Under such conditions, the account for electronic excitation becomes necessary for the modeling of hightemperature gas mixture flows. While the role of rotational and vibrational degrees of freedom in the energy transfer is widely studied in the literature (see Refs. 1-3 and references therein), the influence of electronic excitation of molecules and, especially, atoms on the transport phenomena is not understood until now. Moreover, transport processes are often modeled in a simplified way in terms of constant Prandtl and Schmidt numbers, which are indeed nonconstant in the relaxation zone behind strong shock waves. ${ }^{4}$

Transport properties of ionized gases have received much attention in the literature for the case of chemically equilibrium plasmas. ${ }^{5-17}$ However, in the majority of studies (except Refs. 10 and 12-14) atomic species are considered as structure-less particles. Under this assumption, no internal heat conductivity and bulk viscosity coefficients appear for atomic species, both neutral and ionized, which can cause the loss of accuracy in the transport terms evaluation. Rigorous kinetic theory approaches for transport processes in chemically non-equilibrium ionized flows with electronic degrees of freedom have been proposed recently on the basis of the Chapman-Enskog ${ }^{18-23}$ and $\operatorname{Grad}^{24,25}$ methods. Detailed analysis of thermal conductivity coefficients in electronically excited gases shows an important contribution of electronic levels to the thermal conductivity of both near equilibrium and strongly chemically non-equilibrium plasmas. Nevertheless, studies summarizing the results on electronic excitation for air mixture species and indicating the conditions under which electronically excited states have to be taken into account are still absent.

Another important problem is to understand the role of electronic degrees of freedom in mass and heat transfer in real gas flows. Chemical kinetics, dynamics, and radiation in high-temperature shock heated flows near reentering vehicles have been studied in many papers (see Refs. 2 and 26-32 and many others). However, modeling of convective heat flux in these studies is based on rather simplified models, and no systematic analysis of the influence of electronic excitation on the mass diffusion (MD) and energy transport in real gas flows have been performed until now. 
The objective of the present paper is to generalize the model proposed in Refs. 18-21 and 23 for ionized nonequilibrium flows of air species and their mixtures taking into account the electronic degrees of freedom for all species, both neutral and ionized. Based on the developed model, the transport coefficients: thermal conductivity, shear, bulk and second viscosity, multi-component and thermal diffusion (TD) are then calculated in the temperature range $500-50000 \mathrm{~K}$. The influence of electronic excitation on all transport coefficients is evaluated; the limits of applicability for the Stokes relation are established.

High-temperature strongly non-equilibrium gas flows behind shock waves under conditions of Hermes and Fire II reentry ${ }^{33}$ are studied numerically. On the basis of precomputed fluid-dynamic variables and transport coefficients, we evaluate various contributions to the heat flux in a flow. We consider $N_{2} / N_{2}^{+} / N / N^{+} / e^{-}$and $O_{2} / O_{2}^{+} / O / O^{+} / e^{-}$mixtures, taking into account the rotational, vibrational, and electronic degrees of freedom in molecules and molecular ions as well as electronically excited states in atoms and atomic ions. Dissociation, ionization, and charge transfer reactions are implemented into equations of non-equilibrium chemical kinetics. The sensitivity of the heat flux to electronic excitation and to different chemical reaction rates is examined.

\section{GOVERNING EQUATIONS}

We consider a non-equilibrium high-temperature reacting gas mixture flow under the following relation between characteristic times of collisional processes:

$$
\tau_{\text {tr }}<\tau_{\text {rot }}<\tau_{\text {vibr }}<\tau_{\text {el }} \ll \tau_{\text {react }} \sim \tau_{\text {ioniz }} \sim \tau_{\text {ch-tr }} \sim \theta .
$$

This relation means that relaxation of internal energy, including translational (with the corresponding relaxation time $\left.\tau_{t r}\right)$, rotational $\left(\tau_{r o t}\right)$, vibrational $\left(\tau_{v i b r}\right)$, and electronic $\left(\tau_{e l}\right)$ modes is fast, whereas chemical reactions of dissociation and recombination $\left(\tau_{\text {react }}\right)$, ionization $\left(\tau_{\text {ioniz }}\right)$, and transfer of charge due to particles collisions $\left(\tau_{c h-t r}\right)$ proceed on the macroscopic gas-dynamics time scale $\theta$.

Under such an assumption, the flow is described in the frame of one-temperature kinetic theory approach ${ }^{3}$ and governing equations take the following form:

$$
\begin{gathered}
\frac{d n_{c}}{d t}+n_{c} \nabla \cdot \mathbf{v}+\nabla \cdot\left(n_{c} \mathbf{V}_{c}\right)=R_{c}^{\text {react }}+R_{c}^{\text {ioniz }}+R_{c}^{\text {ch-tr }}, \\
c=1, \ldots, L, \\
\rho \frac{d \mathbf{v}}{d t}+\nabla \cdot \boldsymbol{P}=0, \\
\rho \frac{d U}{d t}+\nabla \cdot \mathbf{q}+\boldsymbol{P}: \nabla \mathbf{v}=0 .
\end{gathered}
$$

Here, $n_{c}$ is the number density of species $c, \mathbf{v}$ is the gas velocity, $L$ is the number of chemical species, $U$ is the total specific energy including that of electronic degrees of freedom, $\mathbf{V}_{c}$ is the diffusion velocity, $\rho$ is the mixture density, $\boldsymbol{P}$ is the pressure tensor, $\mathbf{q}$ is the heat flux, $R_{c}^{\text {react }}, R_{c}^{\text {ioniz }}$ and $R_{c}^{c h-t r}$ are the production terms due to chemical reactions, ionization, and charge-transfer processes, respectively.
The total energy per unit mass in Equation (4) is specified not only by translational, rotational, and vibrational degrees of freedom, as it is basically adopted in nonequilibrium aerothermodynamics, but also by the electronic energy of molecules and atoms

$$
\begin{aligned}
U & =E_{t r}+E_{\text {int }}, \\
\rho E_{t r} & =\sum_{c} \sum_{n, i, j} \int \frac{m_{c} c_{c}^{2}}{2} f_{c, n i j} d \mathbf{u}_{c}, \\
\rho E_{\text {int }} & =\sum_{c} \sum_{n, i, j} \int \varepsilon_{i n t, c}(n, i, j) f_{c, n i j} d \mathbf{u}_{c},
\end{aligned}
$$

where $E_{t r}, E_{\text {int }}$ are the translational and internal energies per unit mass, $f_{c, n i j}$ is the distribution function of chemical species $c$ over velocity $\mathbf{u}_{c}$ and internal energy states $n, i, j$ ( $n$ denotes the electronic energy level, whereas $i$ and $j$ correspond to the vibrational and rotational energies, respectively, for the $n$th electronic state of a molecule), $m_{c}$ is the mass of $c$ th species, and $\mathbf{c}_{c}=\mathbf{u}_{c}-\mathbf{v}$ is its peculiar velocity. The microscopic internal energy for a molecule $\varepsilon_{i n t, m o l}$ or atom $\varepsilon_{i n t, a t}$ is given by

$$
\varepsilon_{i n t, m o l}=\varepsilon_{e l}(n)+\varepsilon_{\text {vibr }}(n, i)+\varepsilon_{r o t}(n, i, j), \quad \varepsilon_{i n t, a t}=\varepsilon_{e l}(n) .
$$

It is worth noting that according to the definition (6), for molecular species all kinds of internal energies are strongly coupled and cannot be considered separately.

The number of accounted electronic energy levels is determined by the so called cut-off criteria, which usually contain the factor of lowering the ionization energy due to the presence of charged particles. ${ }^{34}$ In the present study, we use the criteria suggested in Ref. 35: $\varepsilon_{n}^{c} \leq E_{c}-\Delta E_{c}$, where $E_{c}$ is the ionization energy and the above mentioned lowering factor $\Delta E_{c}$ is set to $1000 \mathrm{~cm}^{-1}$. The electronic energy $\varepsilon_{n}$ and the corresponding statistical weight $g_{n}$ are taken from Ref. 35. The numbers of electronic energy levels for the species considered in the present study are given in Table I.

It is obvious that, if we decrease the lowering factor $\Delta E_{c}$, we include highly located electronic energy levels. Since the number of existing electronic levels close to the ionization energy $E_{c}$ sharply increases, accounting for this great amount of electronic states leads to additional computational difficulties. Retaining high-lying states may increase the total internal energy, and consequently, modify the total specific heat. In the present paper, we consider Boltzmannlike distributions over electronic energy, with a relatively low population of highly located states. Therefore, the intermediate value of $\Delta E_{c}$ has been chosen to avoid numerical difficulties. ${ }^{35}$ On the other hand, it is worth noting that such

TABLE I. Number of electronic energy levels accounted for in the present study.

\begin{tabular}{lccccccccccc}
\hline Species & $N$ & $N^{+}$ & $O$ & $O^{+}$ & $O^{-}$ & $N_{2}$ & $N_{2}^{+}$ & $O_{2}$ & $O_{2}^{+}$ & $N O$ & $N O^{+}$ \\
\hline $\begin{array}{l}\text { Number } \\
\text { of levels }\end{array}$ & 170 & 625 & 204 & 625 & 3 & 5 & 7 & 7 & 7 & 4 & 8 \\
\hline
\end{tabular}


an assumption may lead to an underestimation of the total internal energy for the specific case of an inverse laser, where high-lying electronic states are highly populated.

In high-temperature flows, radiative effects are of crucial importance, and radiative heating becomes comparable to the convective one. In this case, fluid dynamic equations have to be coupled to the equations of radiative heat transfer; advanced models of coupled gas dynamics, chemistry, and radiation are proposed by a number of authors for state-tostate $^{30,36-38}$ and multi-temperature (MT) ${ }^{27-29,32}$ flows (see also references in Ref. 27). Nevertheless, since this study is focused mainly on the effect of electronic excitation on the convective heat flux, we choose the flow conditions under which the contribution of radiative heating does not exceed 5\%-10\% (Refs. 28 and 32), and thus, we do not account for chemical-radiative coupling in the governing equations.

Another important point to be discussed here is accounting for thermal non-equilibrium. It is clear that hightemperature flows in the shock layer are out of thermal equilibrium. In aerospace applications, thermal non-equilibrium is commonly described in the frame of multi-temperature (MT) models by introducing temperatures of different energy modes: rotational $T_{r o t}$, vibrational $T_{v i b r}$, and electronic $T_{e l}$, see Refs. 1-3, 26, 28, 31, and 32 and many other works. Governing equations for these temperatures are usually derived phenomenologically. From the point of view of the rigorous kinetic theory, this procedure is not justified for most cases. Indeed, in the kinetic theory, the macroscopic variables are introduced on the basis of collision invariants; corresponding governing equations are derived from the Boltzmann equation on multiplying it by the invariants and integrating over molecular velocities. Derivation is essentially based on the integral theorems: ${ }^{39}$ for the collision invariants, the integral operator vanishes upon averaging, thus providing zero collision-induced production terms in the conservation equations. For strongly non-equilibrium flows, the fluid dynamic variables are associated with the collision invariants of rapid processes; ${ }^{3}$ these invariants cancel only the collision operator of rapid processes; thus, the production terms due to slow (non-equilibrium) processes appear in the fluid dynamic equations. Therefore, if one is willing to derive governing equations for $T_{r o t}, T_{v i b r}$, or $T_{e l}$, the right point to start is to assign microscopic collision invariants to these variables. The only case when it works is the vibrational energy in a system of harmonic oscillators; $\varepsilon_{v i b r}$ is conserved because of strictly resonant VV exchanges. All other internal energy exchanges are far from being resonant; there is no collision invariant corresponding to $T_{r o t}$ and $T_{e l}$. One can certainly introduce $T_{r o t}$ and $T_{e l}$ artificially by averaging the corresponding microscopic energies $\varepsilon_{r o t}, \varepsilon_{e l}$. But since they are not conserved in rapid processes, applying the integral theorems does not yield zero contribution of corresponding averaged integral operators. This should give rise to additional production terms associated with rapid processes in the fluid dynamic equations. However, in all phenomenological multi-temperature models these additional terms never appear.

Since we have some doubts on the applicability of multitemperature models for gases with electronic excitation, we decided to carry out this study in the frame of the onetemperature model, which can adequately describe chemical non-equilibrium. For thermal non-equilibrium, state-to-state models based on the strict conservation, in rapid processes, of particles in each internal state seem to be more appropriate, although computationally expensive. The work on the statespecific transport model for electronically excited gases is now in progress and will be reported in the next paper.

\section{TRANSPORT AND PRODUCTION TERMS}

The transport and production terms in Eqs. (2)-(4) are specified by the particles distribution function over velocities and internal energy states and thus depend on the approximation of the Chapman-Enskog method. ${ }^{3}$ A modification of the Chapman-Enskog method suitable for strongly chemically non-equilibrium partially ionized gases, taking into account the electronic excitation is proposed in Refs. 18, 20, and 22.

In the zero-order approach of the modified ChapmanEnskog method, the distribution function for atomic and molecular species represents the Maxwell distribution over velocity and the Boltzmann distribution on the rotational, vibrational, and electronic energy for molecules and electronic energy for atoms, and non-equilibrium distribution over chemical species

$$
\begin{aligned}
f_{c l}^{(0)} & =\left(\frac{m_{c}}{2 \pi k T}\right)^{\frac{3}{2}} \frac{n_{c} g_{c l}}{Z_{i n t, c}(T)} \exp \left(-\frac{m c_{c}^{2}}{2 k T}-\frac{\varepsilon_{c l}}{k T}\right), \\
l_{a t} & =\{n\}, l_{m o l}=\{n i j\} .
\end{aligned}
$$

Here, $T$ is the temperature, $k$ is the Boltzmann constant, $g_{c l}$ is the statistical weight (for molecules, $g_{n i j}=g_{n} g_{i} g_{j}$ ), and $Z_{\text {int }, c}(T)$ is the equilibrium internal partition function, $Z_{\text {int }, c}$ $=\sum_{l} g_{c l} \exp \left(-\frac{\varepsilon_{c l}}{k T}\right)$.

Due to the Maxwell velocity distribution, the expressions for the zero-order pressure tensor, diffusion velocity, and heat flux are reduced to

$$
\boldsymbol{P}=p \boldsymbol{I}, \quad \mathbf{V}_{c}=\mathbf{q}=0,
$$

where $p$ is the pressure and $\boldsymbol{I}$ is the unit tensor.

The production terms $R_{c}^{\text {react }}, R_{c}^{\text {ioniz }}$, and $R_{c}^{c h-t r}$ in the zeroorder approximation can be written using the mass action law; the zero-order rate coefficients of non-equilibrium processes are the functions of temperature and can be calculated using the Arrhenius law. Details on the reaction rate calculation for the specific mixtures are given in Section V.

The first-order correction to the distribution function $\varphi_{c l}$ depends on the gradients of fluid dynamic variables and contains the unknown functions $\mathbf{A}_{c l}, \mathbf{D}_{c l}, \boldsymbol{B}_{c l}, F_{c l}$, and $G_{c l}$ which can be found from the linear integral equations

$$
\begin{gathered}
f_{c l}=f_{c l}^{(0)}\left(1+\varphi_{c l}\right), \\
\varphi_{c l}=-\frac{1}{n} \mathbf{A}_{c l} \cdot \nabla \ln T-\frac{1}{n} \sum_{d} \mathbf{D}_{c l}^{d} \cdot \mathbf{d}_{d} \\
-\frac{1}{n} \boldsymbol{B}_{c l}: \nabla \mathbf{v}-\frac{1}{n} F_{c l} \nabla \cdot \mathbf{v}-\frac{1}{n} G_{c l},
\end{gathered}
$$


$\mathbf{d}_{c}$ is the diffusive driving force for species $d$ depending on the gradients of molar fractions and pressure. Integral equations for the unknown functions as well as the constraints providing the uniqueness of their solution are given in Ref. 3.

The transport terms in the first-order (viscous) approximation take the form

$$
\begin{gathered}
\boldsymbol{P}=\left(p-p_{r e l}-\zeta \nabla \cdot \mathbf{v}\right) \boldsymbol{I}-2 \eta \boldsymbol{S}, \\
\mathbf{V}_{c}=-\sum_{d} D_{c d} \mathbf{d}_{c}-D_{T c} \nabla \ln T, \\
\mathbf{q}=-\lambda^{\prime} \nabla T-p \sum_{c} D_{T_{c}} \mathbf{d}_{c}+\sum_{c} \rho_{c} h_{c} \mathbf{V}_{c} .
\end{gathered}
$$

Here, $\boldsymbol{S}$ is the deformation rate tensor; $h_{c}$ is the specific enthalpy of species $c ; \eta, \zeta$ are the shear and bulk viscosity coefficients; $p_{r e l}$ is the relaxation pressure; $D_{c d}, D_{T c}$ are the multi-component diffusion and thermal diffusion coefficients; and $\lambda^{\prime}$ is the partial thermal conductivity coefficient. All transport coefficients are expressed in terms of the bracket integrals of the unknown functions ${ }^{3}$

$$
\begin{aligned}
\eta & =\frac{k T}{10}[\boldsymbol{B}, \boldsymbol{B}], \quad \zeta=k T[F, F], \quad p_{r e l}=k T[F, G], \\
\lambda^{\prime} & =\frac{k}{3}[\mathbf{A}, \mathbf{A}], \quad D_{c d}=\frac{1}{3 n}\left[\mathbf{D}^{c}, \mathbf{D}^{d}\right], \quad D_{T c}=\frac{1}{3 n}\left[\mathbf{D}^{c}, \mathbf{A}\right] .
\end{aligned}
$$

In the above expressions, the bracket integrals are specified by the cross sections of rapid processes (translational and internal energy transitions).

One can see that the normal mean stress includes the terms $\zeta \nabla \cdot \mathbf{v}$ and $p_{r e l}$ associated, respectively, to the flow compressibility and to the contribution of non-equilibrium chemical reactions, ionization, and charge-transfer. Note that in gases with electronic excitation, the bulk viscosity coefficient and relaxation pressure are not equal to zero for atomic species, since there is a channel of internal energy relaxation through the electronic energy transitions between different excited states. It is worth mentioning that in the first-order approximation of the modified Chapman-Enskog method, the production terms $R_{c}^{\text {react }}, R_{c}^{\text {ioniz }}$, and $R_{c}^{c h-t r}$ include the cross-coupling terms similar to those in the normal mean stress; the rate coefficients depend not only on the temperature but also on the species densities $n_{c}$, velocity divergence $\nabla \cdot \mathbf{v}$, and affinities of all reactions in a mixture. ${ }^{40}$ Calculation of these cross-coupling terms is rather complicated, see details in Refs. 41 and 42, and is not discussed in the present study.

The partial thermal conductivity coefficient $\lambda^{\prime}$ describes the transfer of translational and internal energies and can be represented in the following form:

$$
\lambda^{\prime}=\lambda_{\text {tr }}+\lambda_{\text {int }} .
$$

Once again, for atomic species with electronic excitation, the coefficient $\lambda_{\text {int }}$ is not zero. Moreover, under hightemperature conditions, it becomes greater than $\lambda_{t r}$ (see discussion in Section IV).
While studying the diffusion and heat transfer, it is interesting to understand the role of different dissipative processes. For this purpose, we can, similar to Refs. 43-45, express the diffusive and heat flux as a sum of several contributions. In our case, the diffusion velocity is specified by the mass diffusion (MD) and thermal diffusion (TD)

$$
\begin{aligned}
\mathbf{V}_{c} & =\mathbf{V}_{c, M D}+\mathbf{V}_{c, T D}, \\
\mathbf{V}_{c, M D} & =-\sum_{d} D_{c d} \mathbf{d}_{c}, \quad \mathbf{V}_{c, T D}=-D_{T c} \nabla \ln T,
\end{aligned}
$$

whereas the heat flux is determined by three contributions: the Fourier flux associated to thermal conductivity $\mathbf{q}_{F}$, and fluxes due to the mass diffusion $\mathbf{q}_{M D}$ and the thermal diffu$\operatorname{sion} \mathbf{q}_{T D}$

$$
\begin{aligned}
\mathbf{q} & =\mathbf{q}_{F}+\mathbf{q}_{T D}+\mathbf{q}_{M D}, \\
\mathbf{q}_{F} & =-\lambda^{\prime} \nabla T, \quad \mathbf{q}_{T D}=-p \sum_{c} D_{T_{c}} \mathbf{d}_{c}+\sum_{c} \rho_{c} h_{c} \mathbf{V}_{c, T D}, \\
\mathbf{q}_{M D} & =\sum_{c} \rho_{c} h_{c} \mathbf{V}_{c, M D} .
\end{aligned}
$$

The role of different terms in the convective heating behind the shock wave is discussed in Section VI.

\section{TRANSPORT COEFFICIENTS}

In order to calculate the transport coefficients, the unknown functions $\mathbf{A}_{c l}, \mathbf{D}_{c l}, \boldsymbol{B}_{c l}, F_{c l}$, and $G_{c l}$ are expanded into the series of the Sonine polynomials in the reduced peculiar velocity and Waldmann-Trübenbacher polynomials in the total specific energy. ${ }^{3,46}$ It is worth noting that in the present study, under the assumption stated in (6), WaldmannTrübenbacher polynomials may only be taken into account as polynomials in strongly coupled total specific energy. For ionized gas, due to a slow convergence of Sonine polynomials, the expansion includes not only the first non-vanishing terms but also several high-order expansion terms. In the present study, the following terms are taken into account ( $r$ corresponds to the Sonine polynomials, $p$ to the WaldmannTrübenbacher ones): $r=p=0 ; r=1, p=0 ; r=2, p=0$; $r=0, p=1$. Substituting the expansions into the integral equations for functions $\mathbf{A}_{c l}, \mathbf{D}_{c l}, \boldsymbol{B}_{c l}, F_{c l}$, and $G_{c l}$, we derive the linear algebraic equations systems for the expansion coefficients $a_{c, r p}, d_{c, r}^{d}, b_{c, r}, f_{c, r p}$, and $g_{c, r p}$. The transport coefficients are then expressed in terms of the expansion coefficients

$$
\begin{gathered}
\lambda^{\prime}=\sum_{c} \frac{5 k}{4} \frac{n_{c}}{n} a_{c, 10}+\sum_{c} \frac{m_{c}}{2} \frac{n_{c}}{n} c_{i n t, c} a_{c, 01}, \\
D_{c d}=\frac{1}{2 n} d_{c, 0}^{d} \\
D_{T_{c}}=-\frac{1}{2 n} a_{c, 0} \\
\eta=\frac{k T}{2} \sum_{c} \frac{n_{c}}{n} b_{c, 0}, \\
\varsigma=-k T \sum_{c} \frac{n_{c}}{n} f_{c, 10},
\end{gathered}
$$




$$
p_{r e l}=-k T \sum_{c} \frac{n_{c}}{n} g_{c, 10}
$$

$c_{\text {int }, c}$ is the internal specific heat of $c$ species at constant volume. For molecules, $c_{i n t}$ includes the specific heat of electronic, rotational, and vibrational degrees of freedom, and for atoms that of the electronic degrees of freedom.

The linear transport systems for the expansion coefficients contain the bracket integrals as the coefficients. ${ }^{3,46}$ The bracket integrals can be simplified using the Mason and Monchick assumptions ${ }^{47}$ and expressed via reduced collision integrals $\Omega_{c d}^{(l, r) *}$ and integrals depending on the variation of internal energy in inelastic collisions $\Delta \varepsilon=\varepsilon_{k}^{\prime}+\varepsilon_{l}^{\prime}-\varepsilon_{k}-\varepsilon_{l}$ (primes correspond to post-collision internal energies).

For all types of interactions, neutral-neutral, neutralcharged, and charged-charged particles, the reduced collision integrals $\Omega_{c d}^{(l, r) *}$ are calculated using the data reported in Ref. 48. Note that the size of excited particles increases considerably for high states, ${ }^{49,50}$ especially for electronically excited atoms. The influence of atomic and molecular diameters on the transport coefficients was discussed recently in Refs. 16 and 22 for atoms, and in Ref. 51 for molecules. While rovibrational excitation does not noticeably affect the corresponding collision integrals, electronic excitation may lead to a considerable increase in $\Omega_{c d}^{(l, r)}$. This effect is, however, out of the scope of the present study; we plan to investigate it in the future work.

The integrals depending on $\Delta \varepsilon$ are required for the calculation of bulk viscosity, relaxation pressure, and first-order corrections to the reaction rates. For their calculation, it is necessary to introduce some additional assumptions. For neutral atoms with electronically excited states, these integrals can be approximated in terms of the deactivation rate coefficient $k_{10}$ for the transition between the first and ground electronic states. ${ }^{19}$ In our calculations, the deactivation rate coefficient $k_{10}$ was taken from Refs. 52 and 53: $k_{10 N}$ $=10^{-19} \mathrm{~m}^{3} / \mathrm{s} ; \quad k_{10 O}=8 \times 10^{-18} \mathrm{~m}^{3} / \mathrm{s}$. For neutral molecules, the integrals including $\Delta \varepsilon$ can be expressed in terms of the rotational relaxation time. ${ }^{20}$ For ionized atomic species, we have not found the data on $k_{10}$ in the literature, and therefore, we do not include here the results on the bulk viscosity coefficient of atomic ions.

Finally, having the expressions for the bracket integrals one can solve the transport linear algebraic equations using the Gauss method or the more efficient iterative algorithms. ${ }^{46}$

The transport coefficients were calculated for the hightemperature air species in the temperature range $500-50000 \mathrm{~K}$ using the approach developed above. We consider the following atomic species: $\mathrm{N}, \mathrm{N}^{+}, \mathrm{O}, \mathrm{O}^{+}, \mathrm{O}^{-}$, and molecular species: $\mathrm{N}_{2}, \mathrm{~N}_{2}^{+}, \mathrm{O}_{2}, \mathrm{O}_{2}^{+}, \mathrm{NO}, \mathrm{NO}^{+}$.

For the model validation, a comparison with the experimental results or other calculations is desirable. One can find the data on the transport coefficients of equilibrium plasmas for different temperatures and pressures in Refs. 8, 16, and 54-56, respectively. However, direct quantitative comparisons with these results are not feasible. In our approach, the mixture composition is assumed as strongly non-equilibrium, and thus, it is impossible to introduce the reactive thermal conductivity as done in Refs. 57 and 58 and later applied in the studies of equilibrium plasmas. In our model, the energy transfer due to chemical reactions is described in terms of mass and thermal diffusion and cannot be attributed to the reactive heat conductivity. For the validation, we have decided to evaluate species thermodynamic properties. In particular, for all considered species, the specific heat at constant pressure $c_{p}$ was calculated and compared with $c_{p}$ reported in Ref. 35. It is found that for $c_{p}$, the discrepancy is within $0.01 \%-4 \%$ which validates using our scheme in further calculations.

The influence of electronic excitation on the thermal conductivity coefficient $\lambda^{\prime}$ of neutral and ionized atomic species in the temperature range $500-50000 \mathrm{~K}$ is shown in Fig. 1. While the translational heat conductivity coefficient is monotonic with $T$, the total thermal conductivity coefficient $\lambda^{\prime}$ is non-monotonic; for neutral atomic species, the maximum is located about $15000-30000 \mathrm{~K}$, and for ionized species, it is shifted towards higher temperatures, 30000-50000 K. The maximum value of $\lambda^{\prime}$ for neutral species is up to 2.5 higher
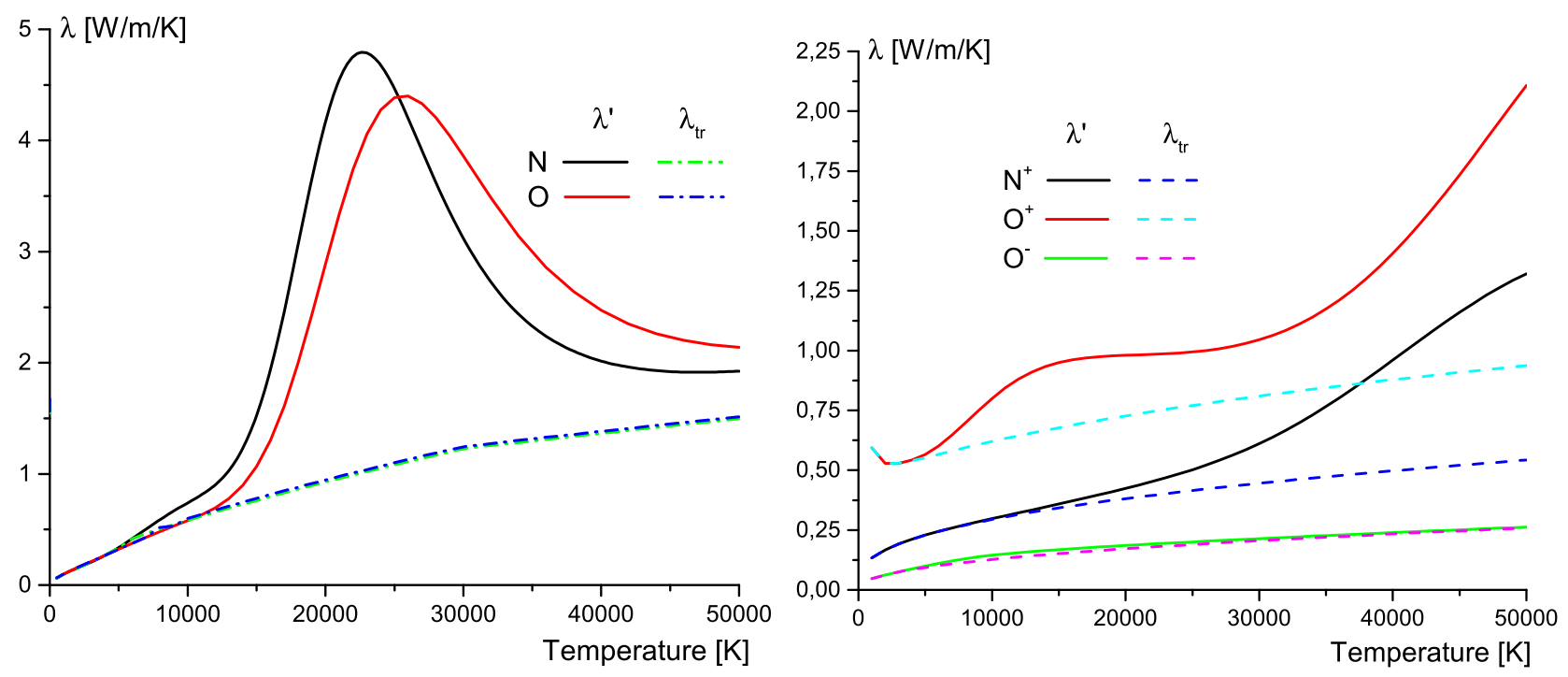

FIG. 1. Thermal conductivity coefficient $\lambda^{\prime}$ of neutral and ionized atomic species as a function of T: contribution of the translational and internal modes. 

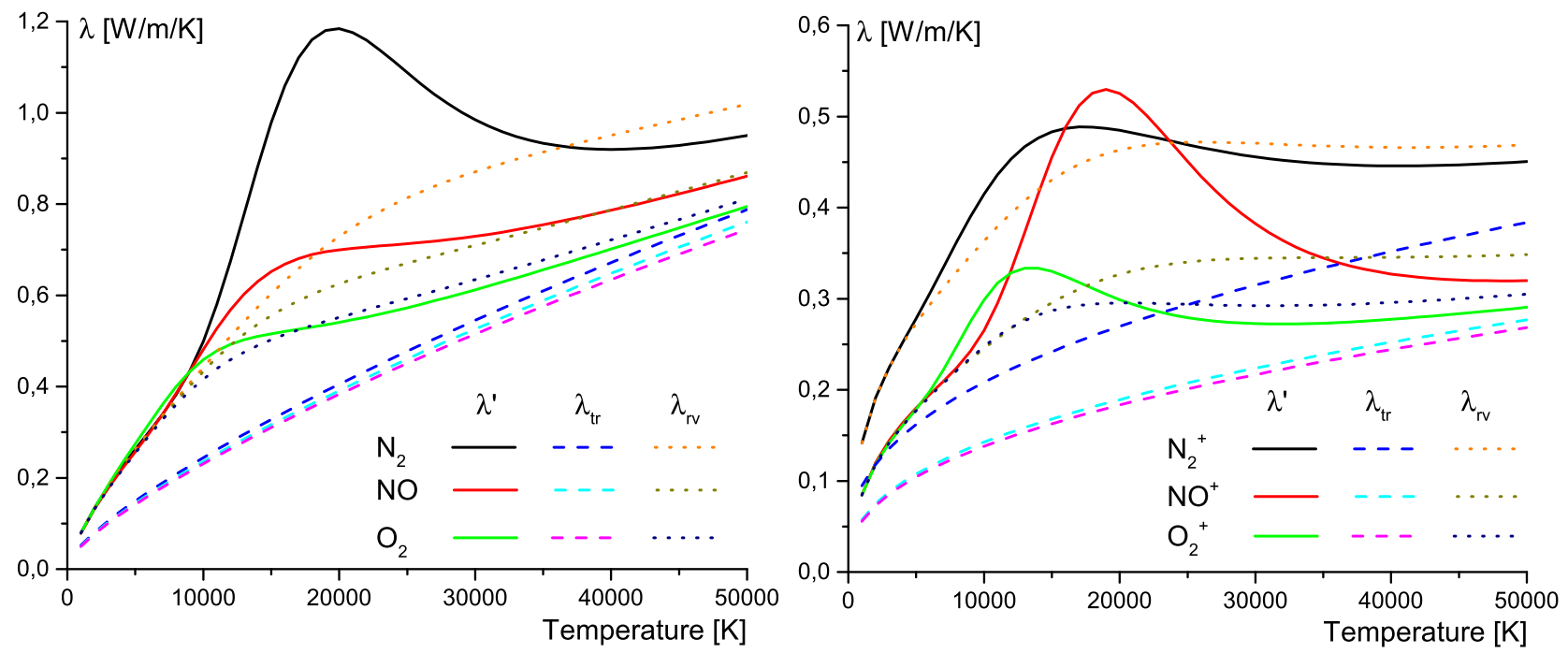

FIG. 2. Thermal conductivity coefficient $\lambda^{\prime}$ of neutral and ionized molecular species as a function of T: contribution of the translational and rotationalvibrational and electronic modes.

than that for ionized atoms. Thus, we can conclude that for atomic species internal thermal conductivity may considerably exceed the translational one, and the most important contribution can be assigned to electronically excited neutral atoms.

In Fig. 2, the thermal conductivity coefficient $\lambda^{\prime}$ of neutral and ionized molecular species is presented in the temperature range $500-50000 \mathrm{~K}$ with the separation of different contributions: $\lambda_{t r, c}$ (taking into account only the translation energy), $\lambda_{\text {int }, c}$ (accounting for the total internal energy), and $\lambda_{r v, c}$ (only rotational-vibrational energy of the ground electronic state is taken into account). Similar to atomic species, the excitation of electronic degrees of freedom substantially influences the thermal conductivity. Neglecting the electronic states results in significant underestimates in the molecular internal thermal conductivity: for the temperature range $15000-20000 \mathrm{~K}$, the coefficient $\lambda_{\text {int }}$ is up to twice larger than the coefficient $\lambda_{r v}$ (for $\mathrm{N}_{2}$ and $\mathrm{NO}^{+}$) calculated, neglecting the electronic states. It is worth noting that in the temperature range $30000-50000 \mathrm{~K}$ for molecular species,

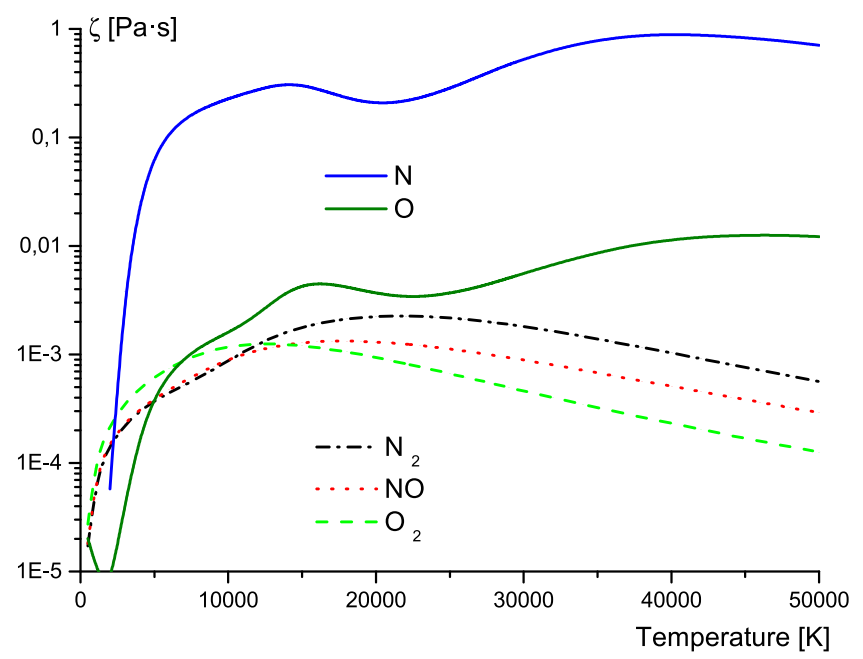

$\lambda_{r v}$ is slightly larger than $\lambda_{\text {int }}$ due to the similar behavior of the specific heat at constant pressure $c_{p}$ (see discussion in Ref. 18). Thus, one can see that the electronic degrees of freedom substantially influence the thermal conductivity, especially for neutral atomic species and $\mathrm{N}_{2}$ and $\mathrm{NO}^{+}$molecules in the temperature range $12000-35000 \mathrm{~K}$, and for ionized atomic species in the temperature range $30000-50000 \mathrm{~K}$. Therefore, electronic excitation has to be taken into account for the heat transfer evaluation in non-equilibrium high-temperature flows, for instance, in shock heated flows.

The bulk $\zeta$ and shear $\eta$ viscosity coefficients are presented in Fig. 3 for neutral molecular and atomic species. It is seen that for temperatures larger than $5000 \mathrm{~K}$ the bulk viscosity coefficient becomes higher than the corresponding shear viscosity coefficient. While shear viscosity of all species monotonically increases with the temperature and do not depend on the electronic degrees of freedom, the bulk viscosity coefficient displays strongly non-monotonic behaviour and depends on the temperature and chemical species: for molecular species, coefficients $\zeta$ have maxima in the

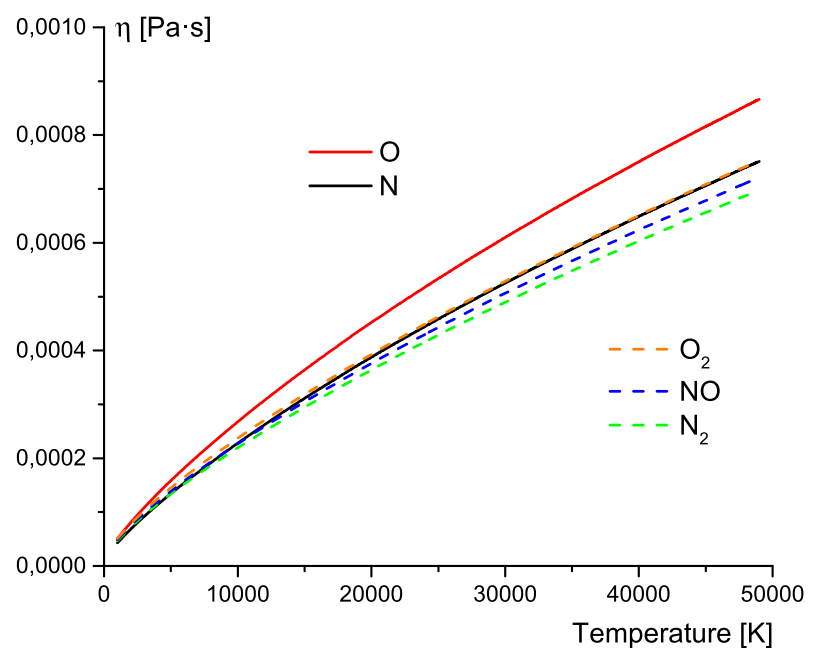

FIG. 3. Bulk viscosity $\zeta$ and shear viscosity $\eta$ coefficients of neutral species as functions of T. 

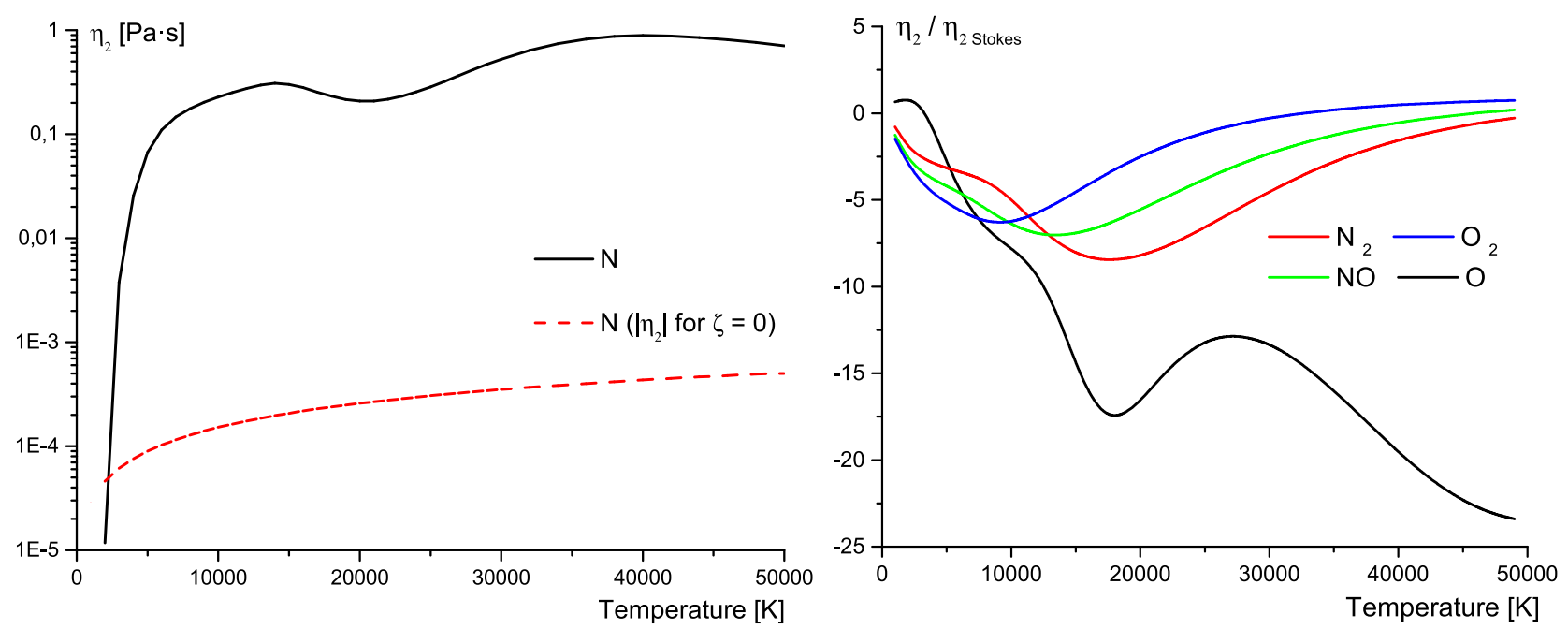

FIG. 4. Second viscosity coefficient $\eta_{2}$ as a function of T for $N$; and the ratio $\eta_{2} / \eta_{2 S t o k e s}$ for other species.

temperature range $15000-20000 \mathrm{~K}$ and then decrease slowly, but for atoms they have local maxima in the same temperature range as molecules and then another one in the range of $35000-50000 \mathrm{~K}$. It is interesting to note that the bulk viscosity coefficient for $N$ is much higher than the corresponding value for $O$. This discrepancy is caused by different deactivation rate constants; $k_{10}$ is two orders of magnitude lower for $N$ than for $O$.

In fluid dynamics, the Stokes relation ${ }^{59}$ connecting the shear $(\eta)$ and the second viscosity $\left(\eta_{2}\right)$ coefficients is commonly adopted: $3 \eta_{2}+2 \eta=0$. It helps in the prediction of flow parameters, but does not explain experimental results on dispersion and sound absorption in polyatomic gases and liquids. ${ }^{60,61}$ The bulk viscosity coefficient was introduced in Refs. 60 and 61 in the form $\zeta=\eta_{2}+\frac{2}{3} \eta$ in order to describe the finite time of energy redistribution between the translational and internal degrees of freedom. For atomic gases, $\zeta$ is commonly assumed to be zero, and therefore $\eta_{2}=-\frac{2}{3} \eta$. In Fig. 4 (left), the second viscosity coefficient $\eta_{2}$ of $\mathrm{N}$ atoms is presented taking into account the non-zero bulk viscosity coefficient $\zeta$ and neglecting it. In the right plot, the ratio of second viscosity coefficients is calculated using our approach and the Stokes formula is given for other species. It is clearly seen that the second viscosity coefficient $\eta_{2}$ does not satisfy the Stokes relation, while for atomic species $\eta_{2}$ increases with the temperature (especially for nitrogen) in the whole temperature range, for molecular species the maximum deviation from the Stokes relation is located in the range $15000-20000 \mathrm{~K}$ (similar to the corresponding $\zeta$ value). Further discussion of the limits of applicability for the Stokes relation can be found in Ref. 62 .

Concluding this section, we would like to emphasize that the effect of electronic excitation on the thermal conductivity, bulk $\zeta$, and second $\eta_{2}$ viscosity coefficients is found to be significant. On the other hand, electronic states do not affect the shear viscosity, multi-component diffusion, and thermal diffusion coefficients (see also Refs. 19 and 20) unless the varying size of excited particles is taken into account. In the latter case, all transport coefficients are considerably reduced at high temperatures.

\section{APPLICATION TO A FLOW BEHIND SHOCK WAVE}

The general model developed above is applied to a nonequilibrium flow of partially ionized nitrogen $\left(\mathrm{N}_{2} / \mathrm{N}_{2}^{+} / \mathrm{N} /\right.$ $\left.\mathrm{N}^{+} / \mathrm{e}^{-}\right)$and oxygen $\left(\mathrm{O}_{2} / \mathrm{O}_{2}^{+} / \mathrm{O} / \mathrm{O}^{+} / e^{-}\right)$mixtures behind a shock wave. Two test cases are considered (TC1 and TC2), corresponding to the specific points of the spacecraft trajectories entering from an interplanetary flight. ${ }^{33} \mathrm{TC} 1$ corresponds to the reentry of European space vehicle Hermes, whereas TC2 corresponds to Fire II experiments (see Table II). For the last fifty years, this latter experiment on the reduced copy of the Apollo space vehicle has been a benchmark for the validation of various thermochemical models. ${ }^{28,29,32,33,63}$ While the vibrational relaxation, chemical reactions, and radiation models were widely assessed, ${ }^{28,32}$ the effect of electronic excitation still remains unstudied (except the work ${ }^{27}$ where highlying electronic energy levels were grouped into one).

In the present study, two mixtures of ionized nitrogen and oxygen have been chosen, first of all, to examine the influence of electronic excitation on the kinetics and heat transfer, and second, to understand the characteristic features of convective heating in different mixtures, including the role of various dissipative processes. Studying these mixtures is for sure useful for further application to real planet atmosphere (for example, for Earth with $78 \%$ of molecular nitrogen and $21 \%$ of oxygen, or for Titan with the atmosphere consisting of $99 \%$ of molecular nitrogen at high altitudes).

We suppose that behind the shock front, the initial lowtemperature mixture consists of neutral molecules. In the shock front, translational, rotational-vibrational, and electronic

TABLE II. Free-stream and post-shock conditions.

\begin{tabular}{lccc}
\hline \hline TC1: Hermes & Velocity $(\mathrm{m} / \mathrm{s})$ & Temperature (K) & Pressure (Pa) \\
\hline Pre-shock & 7198 & 205 & 2 \\
Post-shock & 1207 & 24234 & 1763 \\
TC2: Fire II (1634 s) & Velocity (m/s) & Temperature (K) & Pressure (Pa) \\
\hline Pre-shock & 11360 & 195 & 2 \\
Post-shock & 1899 & 62377 & 3827 \\
\hline \hline
\end{tabular}


degrees of freedom are completely excited, whereas chemical reactions remain frozen. For the heat transfer evaluation, first, the flow-field is simulated in the inviscid Euler approximation providing the macroscopic fluid with dynamic variables and their derivatives, and as a second step, the transport coefficients and the heat flux in a flow are derived applying the transport algorithms as discussed above. Although such a post-processing procedure is approximate, it can give insight to the flow physics avoiding large computational efforts. A similar approach has been justified and used for the heat and mass transfer evaluations in different non-equilibrium flows in Refs. 43-45.

We assume that the flow is steady-state and onedimensional. Under such an assumption, the following set of ordinary differential equations is obtained from the general governing equations:

$$
\begin{gathered}
\frac{d}{d x}\left(v n_{X_{2}}\right)=R_{X_{2}}, \quad X=N, O \\
\frac{d}{d x}\left(v n_{X}\right)=R_{X}, \quad X=N, O \\
\frac{d}{d x}\left(v n_{X_{2}^{+}}\right)=R_{X_{2}^{+}}, \quad X=N, O \\
\frac{d}{d x}\left(v n_{X^{+}}\right)=R_{X^{+}}, X=N, O \\
\frac{d}{d x}\left(v n_{e^{-}}\right)=R_{e^{-}} \\
\frac{d}{d x}\left(p+\rho v^{2}\right)=0 \\
\frac{d}{d x}\left(h+\frac{v^{2}}{2}\right)=0 .
\end{gathered}
$$

Here, $x$ is the distance from the shock front, $h$ is the specific enthalpy depending on $T$ and mixture composition, $R_{c}$ are the production terms due to dissociation, recombination, ionization, and charge-transfer processes

$$
\begin{aligned}
R_{c}^{2 \rightleftharpoons 2} & =\sum_{d, c^{\prime}, d^{\prime}}\left(n_{c^{\prime}} n_{d^{\prime}} k_{c^{\prime} c}^{d^{\prime} d}-n_{c} n_{d} k_{c c^{\prime}}^{d d^{\prime}}\right), \\
R_{c}^{2 \rightleftharpoons 3} & =\sum_{d} n_{d}\left(n_{c^{\prime}} n_{f^{\prime}} k_{r e c, c}^{d}-n_{c} k_{c, d i s s}^{d}\right) .
\end{aligned}
$$

Here, $R_{c}^{2 \rightleftharpoons 2}$ and $R_{c}^{2 \rightleftharpoons 3}$ are the production terms for the reactions within two particles $c$ and $d$, which may lead to the production of two $\left(c^{\prime}\right.$ and $\left.d^{\prime}\right)$ or three new species $\left(c^{\prime}, d^{\prime}\right.$ and $\left.f^{\prime}\right)$ through chemical reactions $(2 \rightleftharpoons 2$ and $2 \rightleftharpoons 3$ ), ionization ( $2 \rightleftharpoons 2$ and $2 \rightleftharpoons 3$ ), and charge-transfer processes ( $2 \rightleftharpoons 2$ only). Production terms due to chemical reactions $R_{c}^{\text {react }}$, ionization $R_{c}^{\text {ioniz }}$, and charge-transfer $R_{c}^{c h-t r}$ in Equation (2) can be expressed via summation of all $R_{c}^{2 \rightleftharpoons 2}$ and $R_{c}^{2 \rightleftharpoons 3}$, which are included in the list of accounted reactions. The dissociation $k_{c, \text { diss }}^{d}$ and exchange $k_{c^{\prime} c}^{d^{\prime} d}$ reaction rate coefficients are calculated using the Arrhenius law. The backward reaction rate coefficients $k_{r e c, c}^{d}$ and $k_{c c^{\prime}}^{d d^{\prime}}$ are obtained using the detailed balance principle.

For considered mixtures, nine reactions are implemented into the kinetic scheme (see Table III). The parameters in the
TABLE III. List of included reactions.

\begin{tabular}{lccc}
\hline \hline No. & $\left(N_{2} / N_{2}^{+} / N / N^{+} / e^{-}\right)$ & References & $\left(O_{2} / O_{2}^{+} / O / O^{+} / e^{-}\right)$ \\
\hline 1 & $N_{2}+N_{2} \rightarrow N+N+N_{2}$ & 64 & $O_{2}+O_{2} \rightarrow O+O+O_{2}$ \\
2 & $N_{2}+N \rightarrow N+N+N$ & 64 & $O_{2}+O \rightarrow O+O+O$ \\
3 & $N_{2}+e^{-} \rightarrow N+N+e^{-}$ & 65 & $O_{2}+e^{-} \rightarrow O+O+e^{-}$ \\
4 & $N_{2}+e^{-} \rightarrow N_{2}^{+}+e^{-}+e^{-}$ & 65 & $O_{2}+e^{-} \rightarrow O_{2}^{+}+e^{-}+e^{-}$ \\
5 & $N+N \rightarrow N^{+}+e^{-}+N$ & 66 & $O+O \rightarrow O^{+}+e^{-}+O$ \\
6 & $N+N \rightarrow N_{2}^{+}+e^{-}$ & 65 & $O+O \rightarrow O_{2}^{+}+e^{-}$ \\
7 & $N_{2}+N^{+} \rightarrow N_{2}^{+}+N$ & 65 & $O+O_{2}^{+} \rightarrow O^{+}+O_{2}$ \\
8 & $N+e^{-} \rightarrow N^{+}+e^{-}+e^{-}$ & 65 & $O+e^{-} \rightarrow O^{+}+e^{-}+e^{-}$ \\
9 & $N_{2}^{+}+N_{2} \rightarrow N^{+}+N+N_{2}$ & 53 & $O_{2}^{+}+O_{2} \rightarrow O^{+}+O+O_{2}$ \\
\hline \hline
\end{tabular}

Arrhenius law are taken from the references indicated in Table III.

For the above reactions, the production terms in Eqs. (23)-(27) take the following form for the nitrogen mixture $\left(N_{2} / N_{2}^{+} / N / N^{+} / e^{-}\right)$:

$$
\begin{aligned}
R_{N_{2}} & =R_{1}+R_{2}+R_{3}+R_{4}+R_{7}, \\
R_{N} & =-2 R_{1}-2 R_{2}-2 R_{3}+R_{5}+2 R_{6}-R_{7}+R_{8}-R_{9}, \\
R_{N_{2}^{+}} & =-R_{4}-R_{6}-R_{7}+R_{9}, \\
R_{N^{+}} & =-R_{5}+R_{7}-R_{8}-R_{9}, \\
R_{e^{-}} & =-R_{4}-R_{5}-R_{6}-R_{8},
\end{aligned}
$$

and for the oxygen mixture $\left(\mathrm{O}_{2} / \mathrm{O}_{2}^{+} / \mathrm{O} / \mathrm{O}^{+} / \mathrm{e}^{-}\right)$:

$$
\begin{aligned}
R_{O_{2}} & =R_{1}+R_{2}+R_{3}+R_{4}-R_{7}, \\
R_{O} & =-2 R_{1}-2 R_{2}-2 R_{3}+R_{5}+2 R_{6}+R_{7}+R_{8}-R_{9}, \\
R_{O_{2}^{+}} & =-R_{4}-R_{6}+R_{7}+R_{9}, \\
R_{O^{+}} & =-R_{5}-R_{7}-R_{8}-R_{9}, \\
R_{e^{-}} & =-R_{4}-R_{5}-R_{6}-R_{8} .
\end{aligned}
$$

We solve Eqs. (23)-(29) using the classical fourth-order Runge-Kutta method. All fluid dynamic parameters $(T, p, \rho$, $v, n_{c}$ ) and their derivatives are obtained as functions of $x$. Then they are used as input for the calculation of transport coefficients (17)-(22) and evaluation of the heat flux and diffusion velocities (16)-(17).

\section{KINETICS AND HEAT TRANSFER BEHIND THE SHOCK WAVE}

For $\left(\mathrm{N}_{2} / \mathrm{N}_{2}^{+} / \mathrm{N} / \mathrm{N}^{+} / \mathrm{e}^{-}\right)$and $\left(\mathrm{O}_{2} / \mathrm{O}_{2}^{+} / \mathrm{O} / \mathrm{O}^{+} / e^{-}\right) \operatorname{mix}-$ tures, under conditions of TC1 and TC2 cases, the flow-field and the heat flux behind the shock wave were calculated. In order to estimate the contribution of electronic excitation to the transport coefficients and heat flux in our simulations, we perform our calculations taking into account the electronically excited states (see Table I) and neglecting them.

\section{A. Flow parameters}

Temperature $T$ as a function of the distance $x$ behind the shock front is presented in Fig. 5 for Hermes (TC1) and Fire II (TC2) test cases. Just behind the shock front, the temperature 

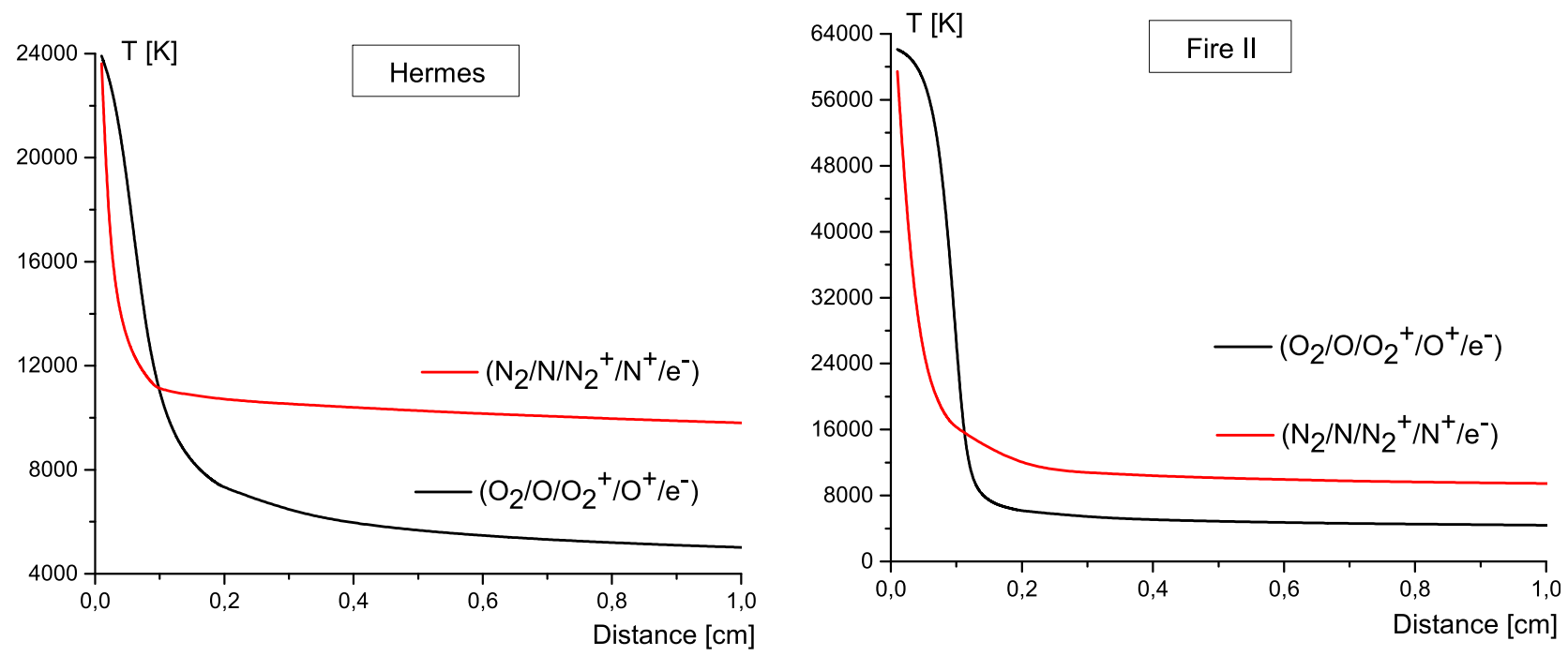

FIG. 5. Temperature $T$ behind the shock wave as a function of $x$ for TC1 (Hermes) and TC2 (Fire II).

drops fast within the distance range $x=0-1.0 \mathrm{~cm}$. For the nitrogen mixture, the results for TC2 practically coincide with those reported in Ref. 30 for Fire II (1634 s) for the air mixture. For nitrogen, the equilibrium temperature is around $12000 \mathrm{~K}$, while for oxygen the equilibrium temperature is lower than $6000 \mathrm{~K}$ and the slope of the curve is different. One can see some delay in the temperature drop for the oxygen mixture, especially for TC2, which can be associated with the dissociation delay obtained while using the rate coefficient of the reaction \#1 given by the McKenzie model. ${ }^{64}$ In Sec. VIC, we will assess other models for the rate coefficient of this reaction and discuss its effect on the heat transfer.

High temperature behind the shock front results in intensive dissociation (see Fig. 6): within the distance range $x=0-1.0 \mathrm{~cm}$ atomic molar fractions sharply increase up to $56 \%$ (TC1) and $79 \%$ (TC2) for nitrogen mixture, and $81 \%$ (TC1) and 92\% (TC2) for oxygen mixture. Surprisingly, just behind the shock front nitrogen dissociates faster than oxygen. This can certainly be attributed to the effect of the rate coefficients of $\# 1$ reaction (see discussion in Sec. VIC). Under specific conditions of TC1 and TC2, the fraction of ionized species considerably increases up to $0.01 \%$ (oxygen) and $0.8 \%$ (nitrogen) for $\mathrm{TC} 1$, and 3\% (oxygen) and $4 \%$ (nitrogen) for TC2 (see Fig. 7). In the nitrogen mixture, ionization plays a slightly greater role; the molar fraction of charged particles in oxygen decreases behind the shock probably due to the electron-impact recombination processes. These results are in line with those reported in Refs. 30 and 34.

Note that in our simulations no influence of electronic excitation on the inviscid flow parameters $\left(T, p, \rho, v, n_{c}\right)$ has been found; the discrepancy does not exceed $0.01 \%$. Therefore, for the inviscid case, electronic degrees of freedom can be neglected in flow-field simulations unless radiation is taken into account. For radiating flows, electronic states can give a considerable contribution to the radiative heat transfer. Moreover, in viscous flows, they can affect the
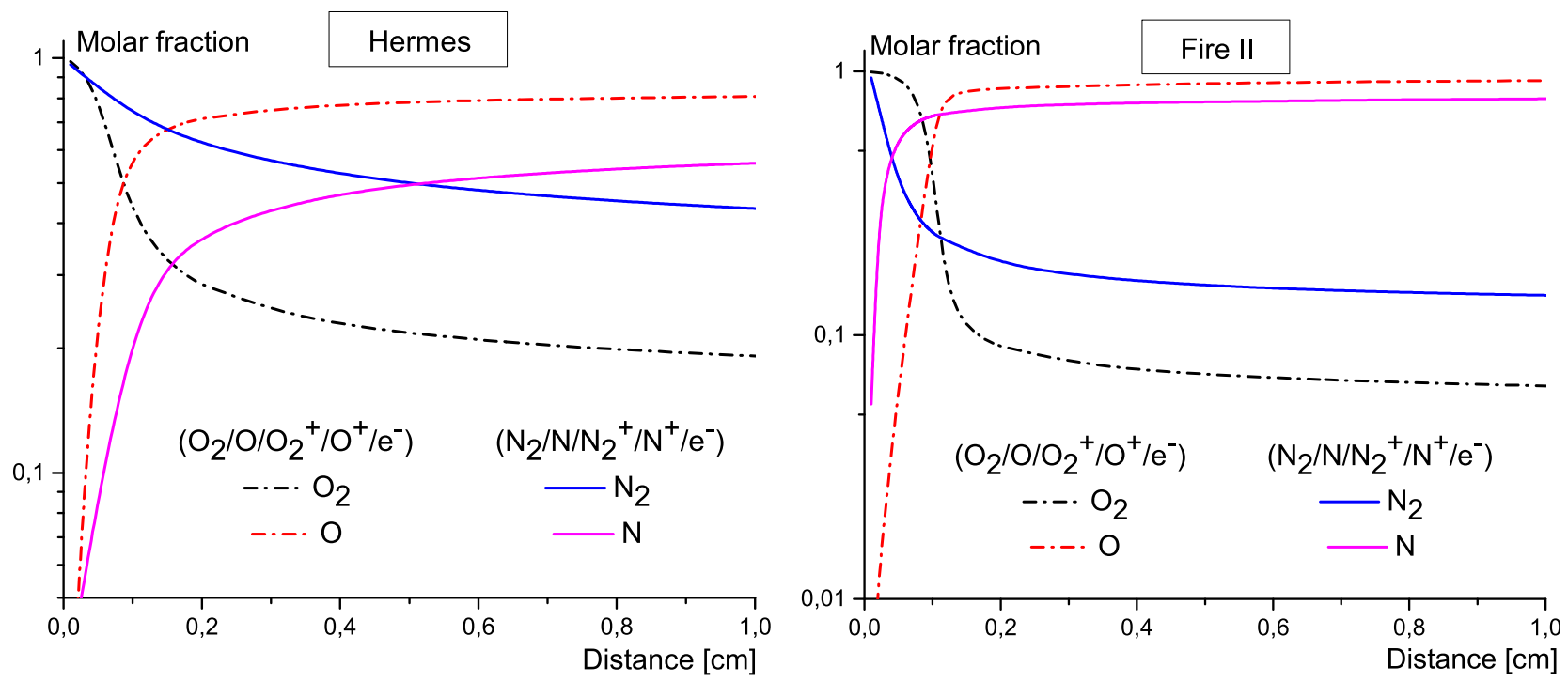

FIG. 6. Molar fractions of neutral species behind the shock wave as functions of $x$ for TC1 and TC2. 

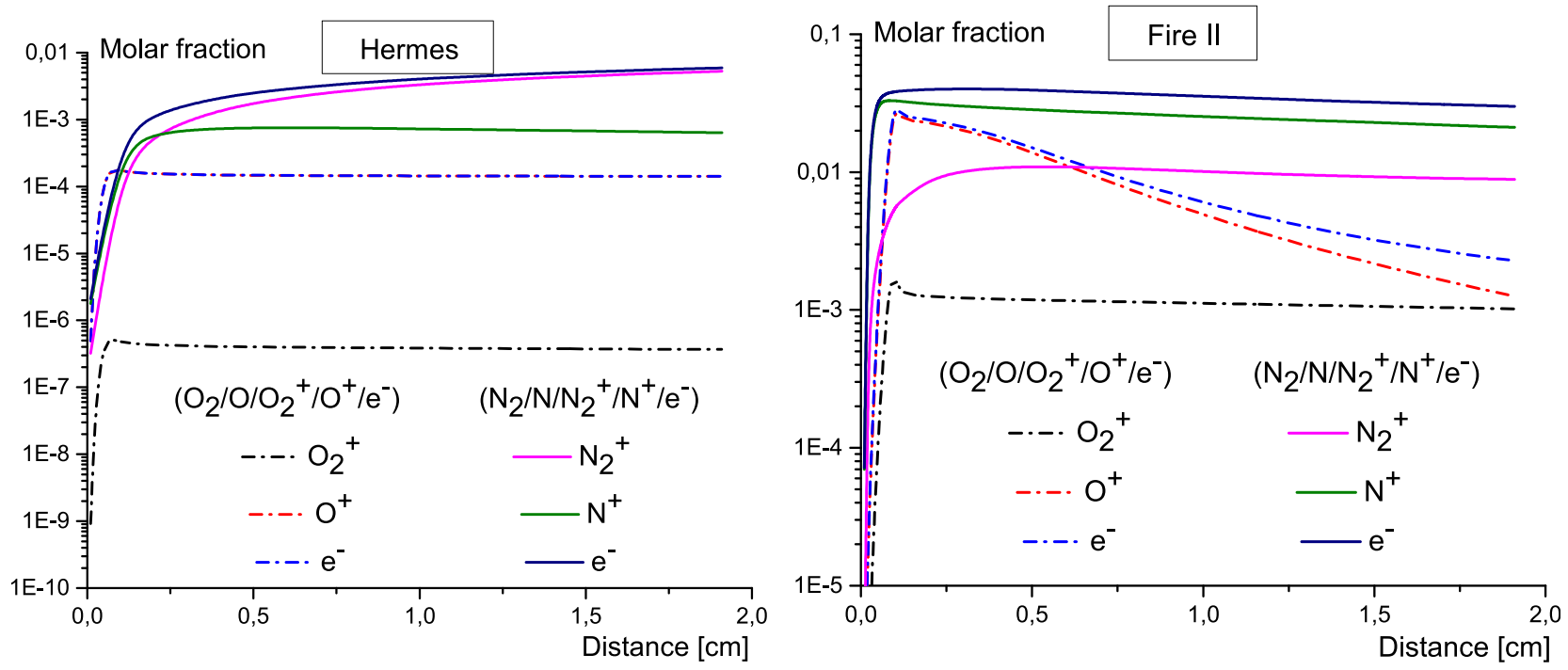

FIG. 7. Molar fractions of charged species behind the shock wave as functions of $x$ for TC1 and TC2.

convective heat flux. This latter effect is studied in Sec. VIB.

\section{B. Transport properties and heat fluxes}

In this subsection, we evaluate the transport properties on the basis of the precalculated flowfield parameters. The total thermal conductivity coefficient $\lambda^{\prime}$ is given in Fig. 8 as a function of $x$ for TC1 and TC2, taking into account the electronically excited states of all the species in the mixture and neglecting the electronic excitation. As shown in Figs. 1 and 2 , the maxima of thermal conductivity coefficients calculated for all the species (both neutral and ionized) taking into account the electronic excitation correspond to the specific conditions of TC1 and TC2 behind the shock front. This explains the fact that in the beginning of the relaxation zone, for the nitrogen mixture the value of $\lambda^{\prime}$ is about two times higher than that calculated neglecting the electronic excitation for both TC1 and TC2; for the oxygen mixture, the influence of electronic states is lower, but is still important at about $30 \%$ (TC1) and 40\% (TC2). With the distance from the shock, the discrepancy between these results decreases, and when the temperature drops lower than $8000 \mathrm{~K}$ for oxygen and $10000 \mathrm{~K}$ for nitrogen, the curves coincide.

In order to assess the proposed model, we compare the convective heat flux obtained using our approach with that calculated in the stagnation point for the Fire II conditions. ${ }^{28,32,63}$ For the considered trajectory point $1634 \mathrm{~s}$, the convective flux varies from 1.5 to $2.2 \mathrm{MW} / \mathrm{m}^{2}$ according to the experiments and simulations. ${ }^{28,32,63}$ This is in line with our results for the near-equilibrium region at a distance $1-2 \mathrm{~cm}$ behind the shock front, where the total heat flux decreases from 3 to $1.5 \mathrm{MW} / \mathrm{m}^{2}$ for the nitrogen mixture, and from 1.5 to $0.3 \mathrm{MW} / \mathrm{m}^{2}$ for the oxygen mixture. Thus, we conclude that our model, even being qualitative due to the applied post-processing procedure, provides a satisfactory
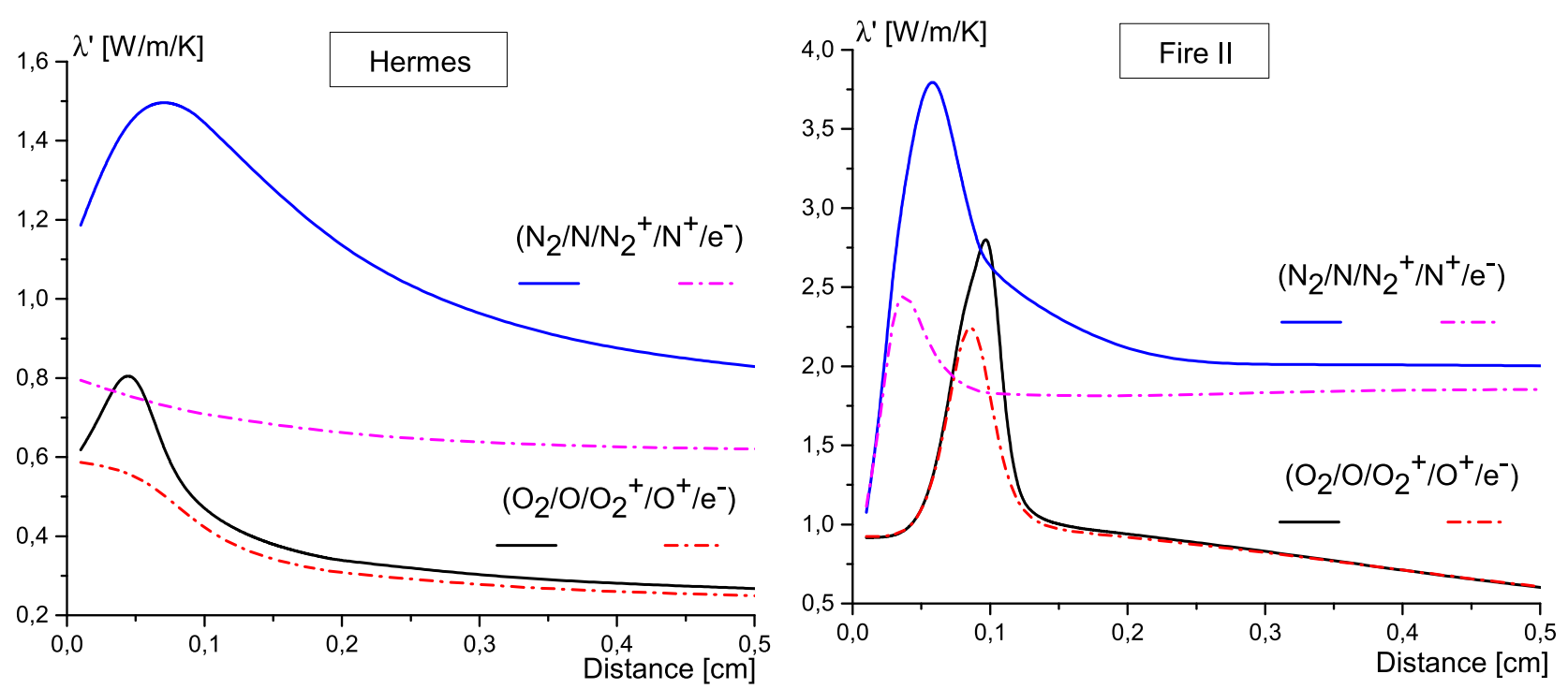

FIG. 8. Thermal conductivity coefficient behind the shock wave as a function of distance $x$ for TC1 and TC2 calculated taking into account the electronic excitation (solid lines) and neglecting it (dashed-dotted lines). 

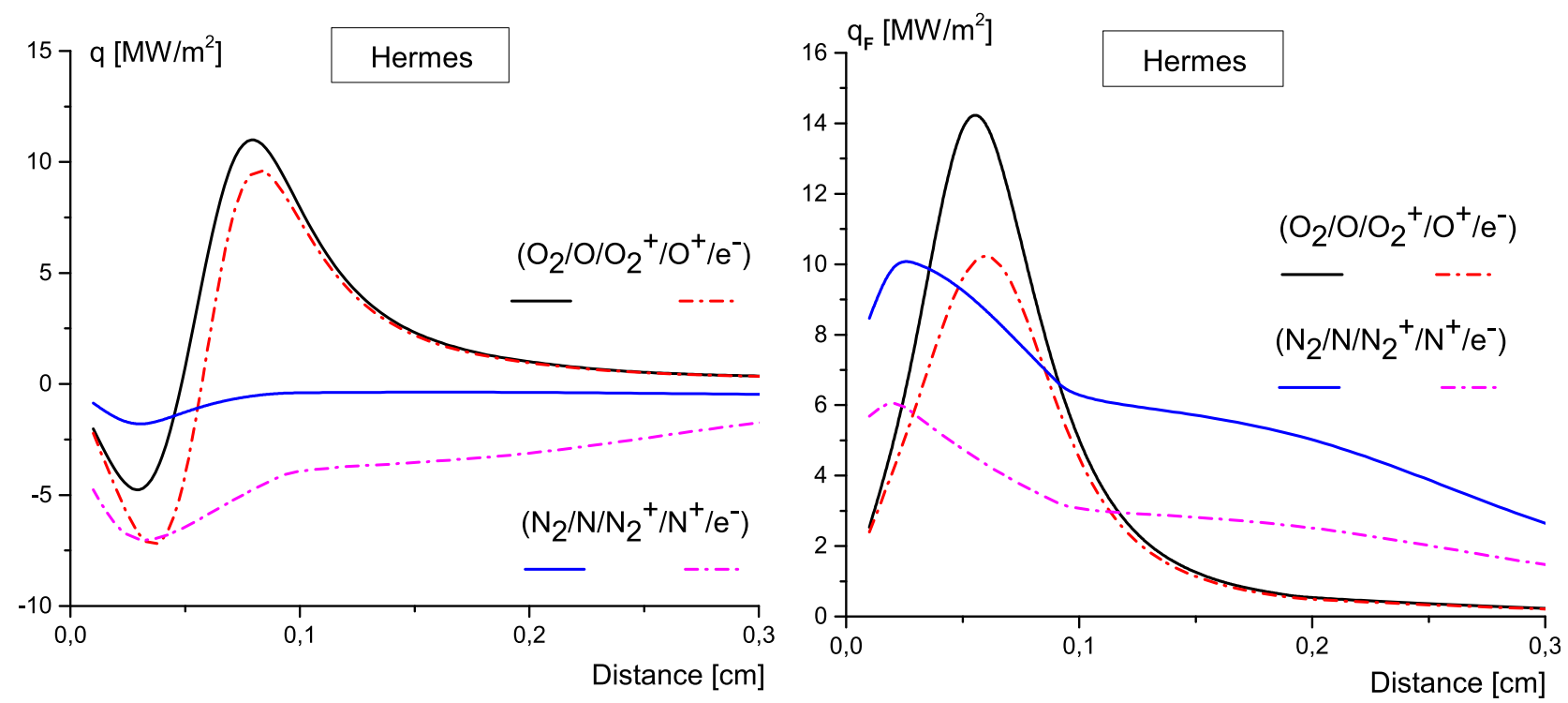

FIG. 9. Total heat flux $\mathbf{q}$ and Fourier flux $\mathbf{q}_{F}$ as functions of $x$ for TC1 calculated taking into account the electronic excitation (solid lines) and neglecting it (dashed-dotted lines).

agreement with the existing experimental and numerical data. It is worth noting that there are many data on the convective heating in the stagnation point; however, the heat flux distributions along the stagnation line starting from the bow shock are basically missing. Our simulations may help to fill this gap.

Now let us examine the total heat flux and the contributions of different dissipative processes to the energy transfer calculated taking into account and neglecting the electronic excitation. These results are presented in Figs. 9 and 10 for TC1 and in Figs. 11 and 12 for TC2. For TC1, it is clearly seen that the electronic excitation plays an important role for the Fourier flux, whereas its effect on the heat transfer due to mass diffusion and thermal diffusion is rather weak (for $\mathbf{q}_{T D}$ this effect can be neglected at all; since the curves with and without electronic excitation coincide, we do not plot them).

Nevertheless, the overall contribution of electronic states to the total heat flux depends significantly on the competition of different dissipative processes involved in the energy transfer; these processes can, in turn, be affected by the electronic excitation or not. While for TC1 all the terms in Eq. (12) are of the same order of magnitude (an important role of the heat flux due to conduction $\mathbf{q}_{F}$ should be emphasized), and a considerable effect of excited electronic states on the total heat flux is observed (especially for nitrogen), for TC2 these terms are considerably different. Since for $\mathrm{TC} 2$ the thermal diffusion plays the dominating role in the total heat transfer (TD is the process which is not affected by electronic excitation), the influence of electronic excitation
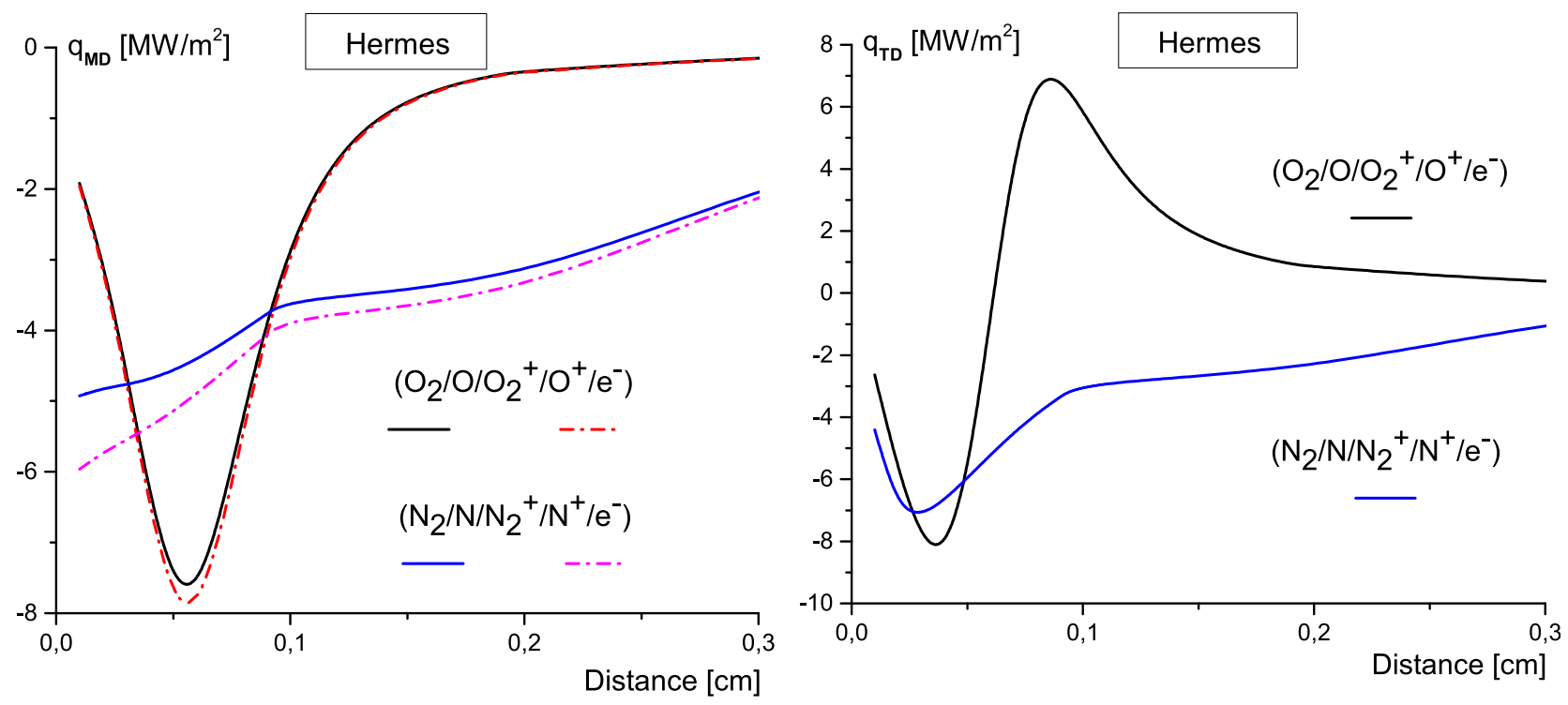

FIG. 10. Heat flux due to mass diffusion $\mathbf{q}_{M D}$ and thermal diffusion $\mathbf{q}_{T D}$ as functions of $x$ for TC1 calculated taking into account the electronic excitation (solid lines) and neglecting it (dashed-dotted lines). 

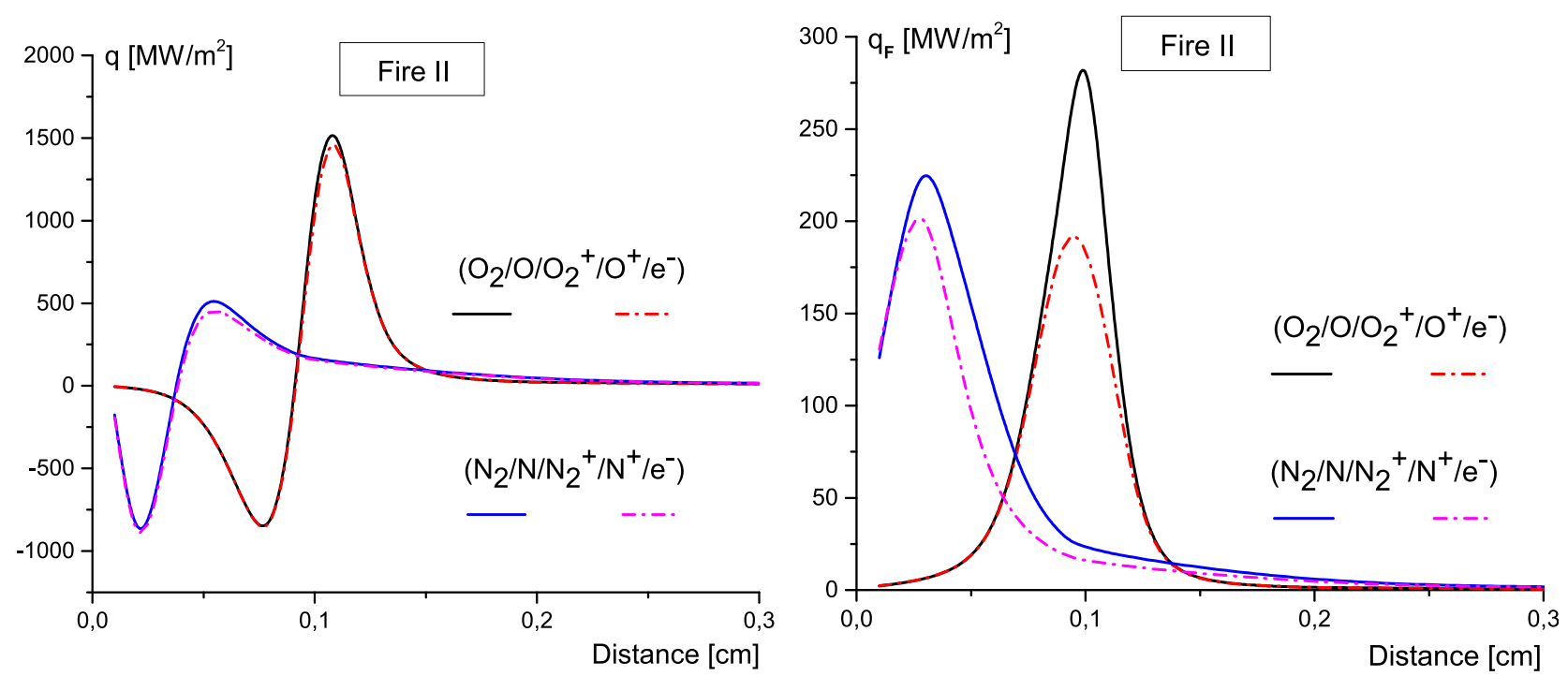

FIG. 11. Total heat flux $\mathbf{q}$ and Fourier flux $\mathbf{q}_{F}$ as a function of distance $x$ for TC2 calculated taking into account the electronic excitation (solid lines) and neglecting it (dashed-dotted lines).

is not so important as for TC1. On the whole, electronic excitation gives a contribution of about $20 \%$ to the total heat flux, but remains higher for the heat conduction and correspondingly for the Fourier flux.

Since all dissipative processes act on a competitive basis on the heat flux, the behaviour becomes essentially nonmonotonic, and moreover, the total heat flux changes its sign. Note that, for the non-ionized mixtures behind the shock front, a similar non-monotonic behaviour of the energy flux is found in Ref. 45 for high Mach numbers. Let us examine in detail each contribution into the heat flux. As the temperature decreases behind the shock front and the thermal conductivity coefficient is always positive, the Fourier flux $\mathbf{q}_{F}$ is positive, too, for any distance behind the shock. The formation of new species due to dissociation and ionization yields their negative diffusive fluxes; since these species carry higher enthalpy, the flux due to mass diffusion $\mathbf{q}_{M D}$ remains negative with $x$. At the same time, the thermal diffusion flux $\mathbf{q}_{T D}$ depends on both temperature and molar fraction gradients, which yields the competition between them, and therefore, $\mathbf{q}_{T D}$ alternates its sign. It is worth noting that the contribution of thermal diffusion to the heat transfer in shock heated non-ionized gases is negligibly weak. ${ }^{45}$ On the other hand, for ionized gases not exposed to the action of the electro-magnetic field, the arising effect of ambipolar diffusion reduces the effect of thermal diffusion. ${ }^{67,68}$ In our case, we do not account for the electromagnetic field, that is why a strong effect of thermal diffusion is found and can be attributed to large values of thermal diffusion coefficients for electrons. ${ }^{4,21}$

\section{The effect of dissociation rate coefficient}

Let us discuss the influence of the reaction rate coefficients on flow parameters and heat transfer. It can be seen from Figs. 5 and 6 that using the McKenzie mode ${ }^{64}$ for the reaction \#1 yields a significant delay of oxygen dissociation just behind the
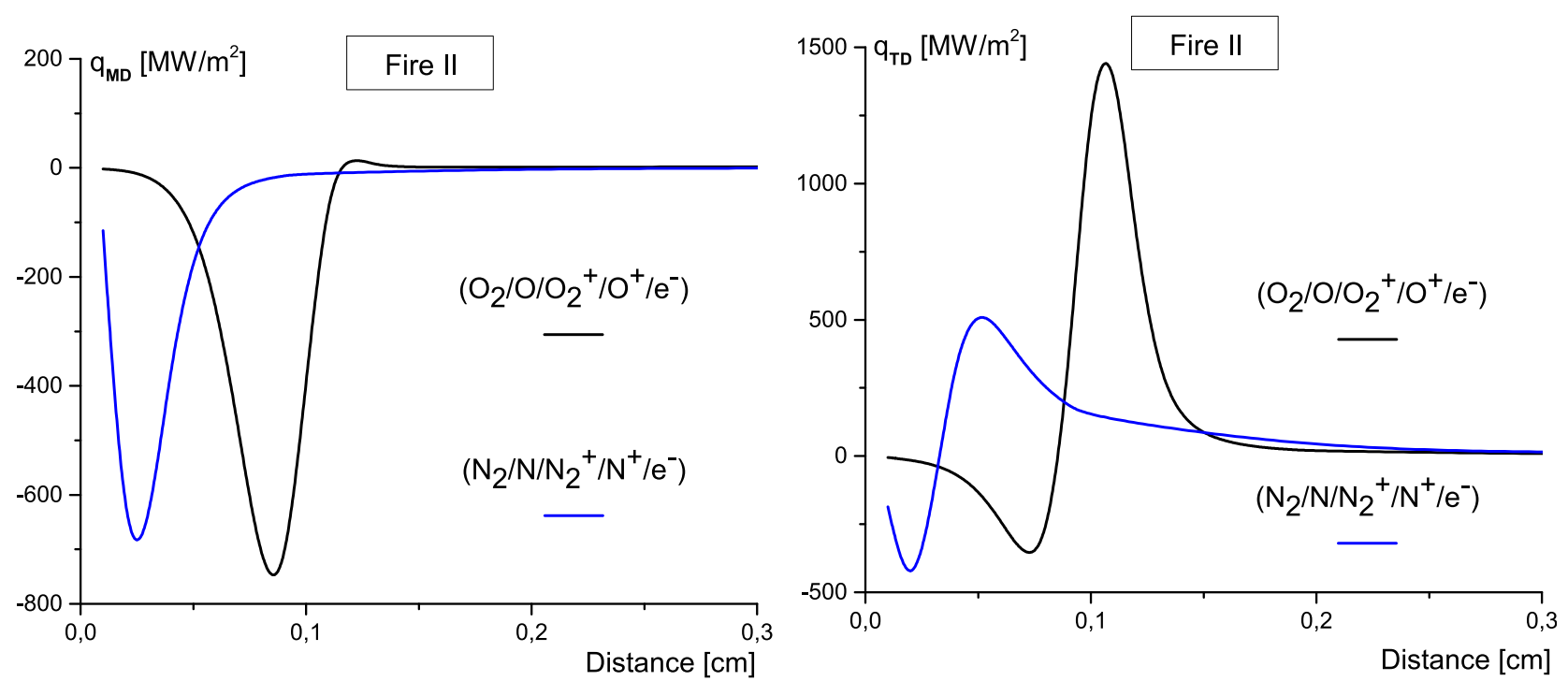

FIG. 12. Heat fluxes due to mass diffusion $\mathbf{q}_{M D}$ and thermal diffusion $\mathbf{q}_{T D}$ as a function of distance $x$ for TC2. 

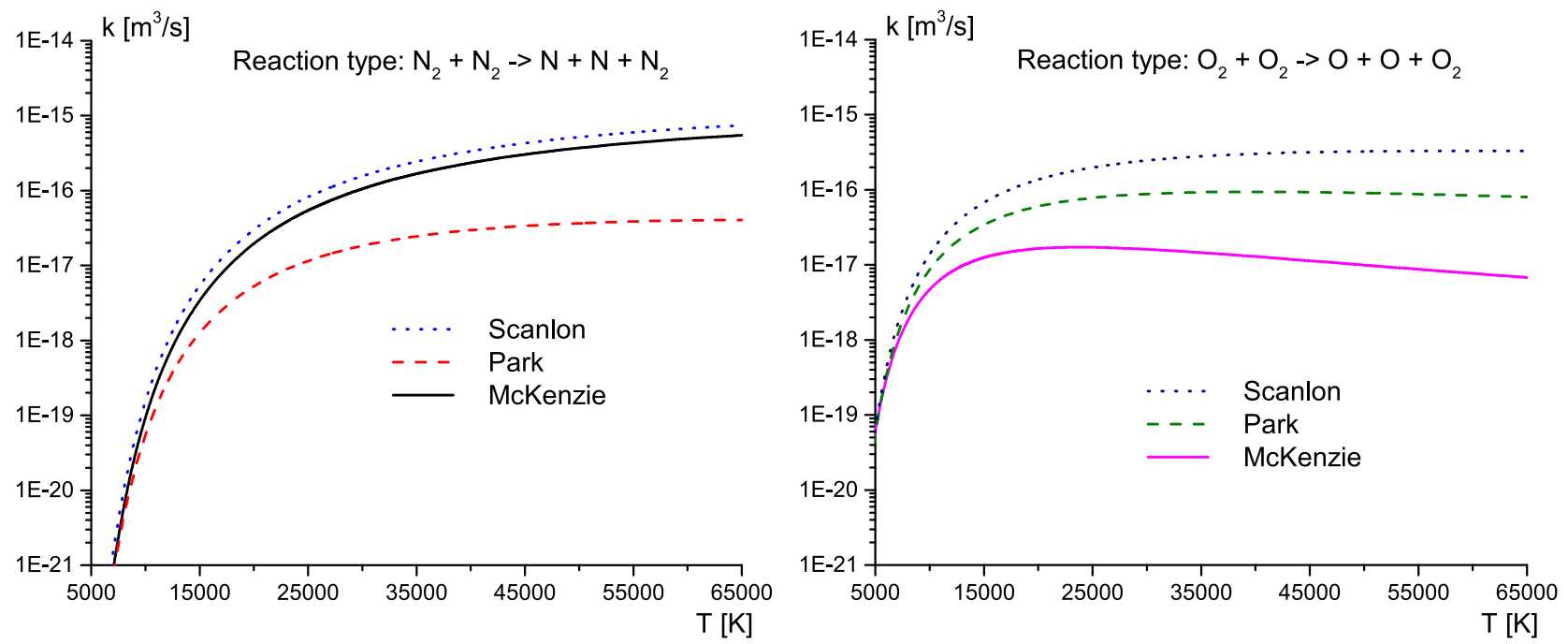

FIG. 13. Reaction rate coefficient $k_{1}$ as a function of $T$ for different models.

shock front, which is quite surprising for high-temperature flows. Indeed, the model ${ }^{64}$ may be not applicable under such high-temperature conditions. For this reason, we assess three different data sets for reaction \#1: the McKenzie model ${ }^{64}$ and the models of Park ${ }^{69}$ and Scanlon. ${ }^{70}$ A comparison of the rate coefficients $k_{1}(T)$ for nitrogen (see Fig. 13) shows no difference between the McKenzie and recent Scanlon models, whereas the Park model in the high-temperature region underestimates the rate coefficients up to two orders of magnitude. At the same time, for oxygen, we see an opposite trend: rather similar dissociation rate coefficients for the Park and Scanlon models versus essentially underestimated data provided by the McKenzie model. Indeed, the latter model gives an unphysical decrease in the dissociation rate coefficients at $T>20000 \mathrm{~K}$.

Since such a great difference is found for the oxygen dissociation reaction \#1, we compare the results for the flowfield and heat fluxes obtained in oxygen for various dissociation models. Using the models ${ }^{69,70}$ yields higher dissociation and lower ionization rates just behind the shock front (see
Fig. 14) and faster temperature drop (Fig. 15). The total heat flux also depends considerably on the dissociation rate modeling (see Fig. 15) and varies up to 1.5 times for different models; its minima and maxima are shifted towards the shock front for the models. ${ }^{69,70}$

Concluding these results we can recommend the use of the Scanlon model $^{70}$ for reaction \#1 at high temperatures, providing a more reliable behavior of the fluid dynamic variables and energy fluxes.

\section{CONCLUSIONS}

A theoretical model of the transport properties in strongly non-equilibrium flows of neutral and ionized atomic and molecular species mixtures with electronic excitation is developed on the basis of the modified Chapman-Enskog method. The contribution of electronic degrees of freedom to thermal conductivity coefficient is found to be important. For atomic species, electronic excitation plays the most
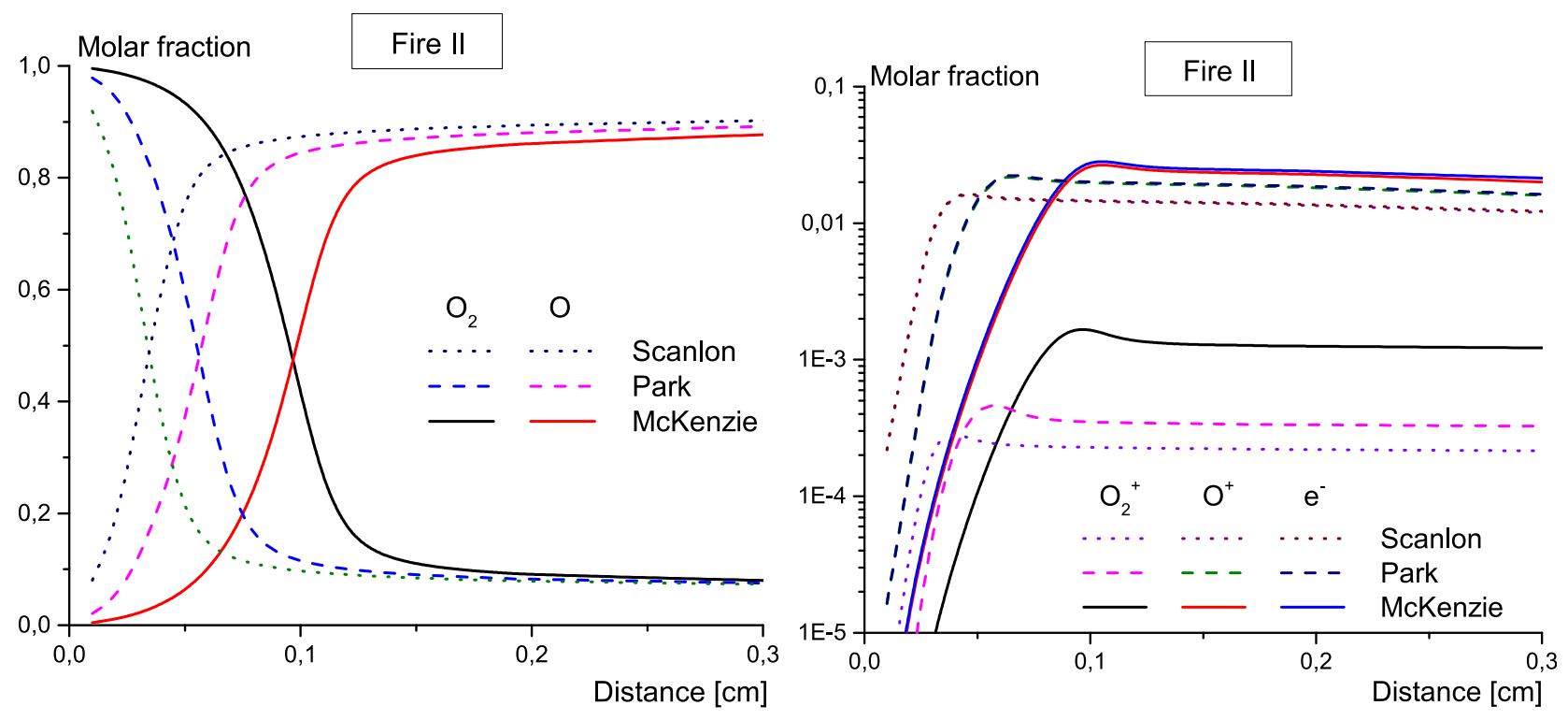

FIG. 14. Molar fractions of neutral and charged species behind the shock wave as functions of $x$ for TC2 calculated using different reaction rate coefficients $k_{1}$. 

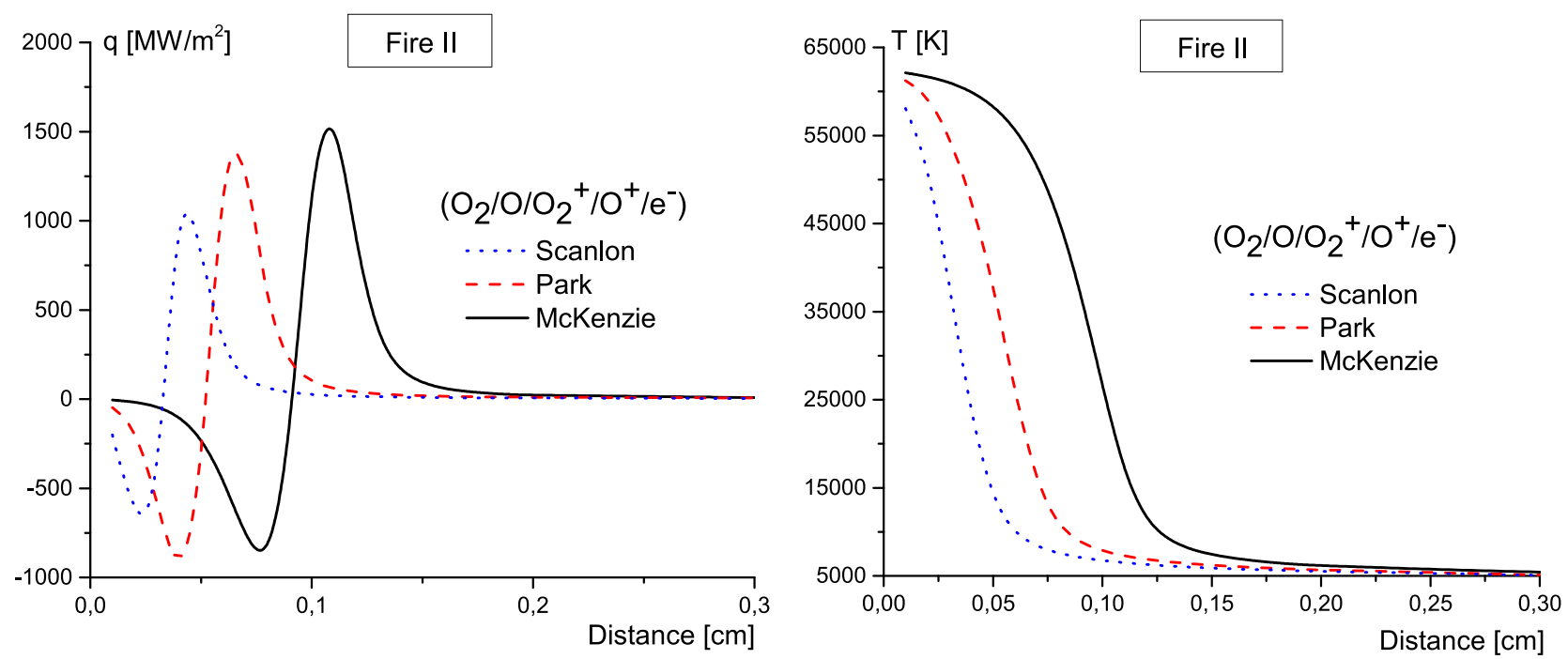

FIG. 15. Temperature $T$ and total heat fluxes $\mathbf{q}$ as a function of distance $x$ for TC2 calculated using different reaction rate coefficients $k_{1}$.

important role in the temperature range $15000-30000 \mathrm{~K}$ for neutral, and $30000-50000 \mathrm{~K}$ for ionized species; under these conditions, the electronic thermal conductivity substantially exceeds the translational one. The maximum value of $\lambda^{\prime}$ for neutral species is up to 2.5 times higher than that for the ionized atoms. Similar to the atomic species, excitation of electronic degrees of freedom considerably influences the thermal conductivity coefficient of molecules: neglecting the electronic states results in the significant underestimation of the molecular thermal conductivity, up to two times.

Bulk viscosity coefficients are also strongly affected by the electronic excitation; it is shown that bulk viscosity of the atomic species is not zero, contrary to the commonly used assumption. On the other hand, shear viscosity, multicomponent diffusion, and thermal diffusion coefficients are not sensible to electronic excitation if one assumes that the size of the excited particles does not depend on the electronic state. The limits of applicability for the Stokes relation are discussed; it is found that at high temperatures, this relation is violated not only for molecular species but also for electronically excited atomic gases.

Two test cases corresponding to the Hermes and Fire II experiments are simulated numerically. Strongly nonequilibrium one-dimensional steady-state flows of chemically reacting nitrogen $N_{2} / N_{2}^{+} / N / N^{+} / e^{-}$and oxygen $\mathrm{O}_{2} / \mathrm{O}_{2}^{+} / \mathrm{O} / \mathrm{O}^{+} / e^{-}$mixtures behind the plane shock wave are studied, taking into account the electronic degrees of freedom of both the neutral and ionized species. No influence of electronic excitation on the inviscid flow parameters is found. Nevertheless, one should keep in mind that under similar conditions, the electronic states may considerably affect the radiative heat flux if chemical-radiative coupling in the flow is taken into account. Moreover, in viscous flows, electronic degrees of freedom substantially influence the thermal conductivity coefficients, and therefore, the Fourier energy flux behind the shock front for temperatures larger than $10000 \mathrm{~K}$ for nitrogen and $8000 \mathrm{~K}$ for oxygen.

The contribution of different dissipative processes into the total heat flux is analyzed. At the beginning of the relaxation zone, for the Hermes case, the contributions of thermal conductivity, mass diffusion, and thermal diffusion to the total heat flux are of the same order for both mixture flows. In this case, the role of electronic degrees of freedom in the heat transfer is found to be important, especially for nitrogen. For the Fire II test case, the thermal diffusion process plays a dominant role in the heat transfer. This process is not sensible to electronic excitation, and therefore, the total heat flux is not affected by the electronic states. It is worth mentioning that since the signs of various heat flux terms are different, their competition leads to a noticeable reduction in the total heat flux in shock heated flows, and thus, weakens the role of electronically excited states in the overall convicted heating.

A strong dependence of flow parameters and heat flux on the chemical reaction rates is found. Comparison of the different models for dissociation rate coefficients shows that the Scanlon model provides a more reliable behavior of fluid dynamic variables, whereas the McKenzie model yields an unexpected delay of oxygen dissociation just behind the shock front and overpredicted heat flux values with the maximum shifted away from the shock front.

Further studies of the role of electronic excitation in the heat transfer should take into account the thermal nonequilibrium and chemical-radiative coupling in a flow. Our work on the state-to-state transport model accounting for atomic and molecular electronic degrees of freedom is in progress.

\section{ACKNOWLEDGMENTS}

This study was supported by the Russian Foundation for Basic Research (Project Nos. 16-38-60009 and 15-08-03371) and Saint Petersburg State University, Project No. 6.37.206.2016.

${ }^{1} \mathrm{R}$. Brun, Transport et relaxation dans les écoulements gazeux (Masson, Paris/New York/Barselone/Milan/Mexico/Saõ Paulo, 1986).

${ }^{2}$ C. Park, Nonequilibrium Hypersonic Aerothermodynamics (John Wiley and Sons, New York/Chichester/Brisbane/Toronto/Singapore, 1990). 
${ }^{3}$ E. Nagnibeda and E. Kustova, Nonequilibrium Reacting Gas Flows. Kinetic Theory of Transport and Relaxation Processes (Springer-Verlag, Berlin, Heidelberg, 2009).

${ }^{4}$ V. Istomin and E. Kustova, in 29th International Symposium on Shock Waves (Springer International Publishing, 2015), Vol. 1, pp. 161-166.

${ }^{5}$ R. Devoto, Phys. Fluids 9, 1230 (1966).

${ }^{6}$ R. Devoto, Phys. Fluids 10, 354 (1967).

${ }^{7}$ S. Vasil'evsky, I. Sokolova, and G. Tirskyi, J. Appl. Mech. Tech. Phys. 27, 68 (1986).

${ }^{8}$ I. Sokolova, Math. Mod. 1, 84 (1989).

${ }^{9}$ A. Murphy and C. Arundell, Plasma Chem. Plasma Process 14, 451 (1994).

${ }^{10}$ M. Capitelli, R. Celiberto, C. Gorse, A. Laricchiuta, D. Pagano, and P. Traversa, Phys. Rev. E 69, 026412 (2004).

${ }^{11}$ T. Magin and G. Degrez, Phys. Rev. E 70, 046412 (2004).

${ }^{12}$ D. Bruno, A. Laricchiuta, M. Capitelli, and C. Catalfamo, Phys. Plasmas 14, 022303 (2007).

${ }^{13}$ D. Bruno, M. Capitelli, C. Catalfamo, and A. Laricchiuta, Phys. Plasmas 14, 072308 (2007).

${ }^{14}$ D. Bruno, M. Capitelli, C. Catalfamo, and A. Laricchiuta, Phys. Plasmas 15, 112306 (2008).

${ }^{15}$ A. Orsini and E. Kustova, Phys. Rev. E 79, 056309 (2009).

${ }^{16}$ M. Capitelli, D. Bruno, and A. Laricchiuta, Fundamental Aspects of Plasma Chemical Physics: Transport, Springer Series on Atomic, Optical, and Plasma Physics Vol. 74 (Springer Verlag, Berlin, 2013).

${ }^{17}$ V. A. Istomin and E. V. Kustova, "Rarefied gas dynamics," AIP Conf. Proc. 1786, 150002 (2016).

${ }^{18}$ E. Kustova and L. Puzyreva, Phys. Rev. E 80, 046407 (2009).

${ }^{19}$ V. Istomin, E. Kustova, and L. Puzyreva, "Rarefied gas dynamics," AIP Conf. Proc. 1333, 667-672 (2011).

${ }^{20}$ V. Istomin and E. Kustova, "Rarefied gas dynamics," AIP Conf. Proc. 1501, 168-174 (2012).

${ }^{21}$ V. Istomin and E. Kustova, "Rarefied gas dynamics," AIP Conf. Proc. 1628, 1221-1228 (2014).

${ }^{22}$ V. A. Istomin, E. V. Kustova, and M. A. Mekhonoshina, J. Chem. Phys. 140, 184311 (2014).

${ }^{23}$ V. A. Istomin and E. V. Kustova, "Rarefied gas dynamics," AIP Conf. Proc. 1786, 150003 (2016).

${ }^{24}$ V. M. Zhdanov and A. A. Stepanenko, Physica A 446, 35 (2016).

${ }^{25}$ V. M. Zhdanov and A. A. Stepanenko, Physica A 461, 310 (2016).

${ }^{26}$ G. Candler and R. MacCormac, AIAA Paper No. 88-0511, 1988.

${ }^{27} \mathrm{C}$. O. Johnston, "Nonequilibrium shock-layer radiative heating for Earth and Titan entry," Ph.D. thesis (Virginia Polytechnic Institute and State University, Virginia, USA, 2006).

${ }^{28}$ C. Johnston, B. Hollis, and K. Sutton, AIAA Paper No. 2007-3908, 2007.

${ }^{29}$ S. Surzhikov, Russ. J. Phys. Chem. B. 2, 814 (2008).

${ }^{30}$ M. Panesi, T. E. Magin, A. Bourdon, A. Bultel, and O. Chazot, J. Thermophys. Heat Transfer 23, 236 (2009).

${ }^{31}$ A. Guy, A. Bourdon, and M.-Y. Perrin, Phys. Plasmas 22, 043507 (2015).

${ }^{32}$ S. Surzhikov, Tech. Phys. 61, 349 (2016).

${ }^{33}$ D. L. Cauchon, "Radiative heating results from FIRE II flight experiment at a re-entry velocity of $11.4 \mathrm{~km} / \mathrm{s}$," Technical Report No. X-1402, NASA, NASA Technical Memorandum, 1967.

${ }^{34}$ M. Capitelli, G. Colonna, and A. D'Angola, Fundamental Aspects of Plasma Chemical Physics: Thermodynamics, Springer Series on Atomic, Optical, and Plasma Physics Vol. 66 (Springer Verlag, Berlin, 2012).

${ }^{35}$ M. Capitelli, G. Colonna, D. Giordano, L. Marraffa, A. Casavola, P. Minelli, D. Pagano, L. Pietanza, and F. Taccogna, Tables of Internal Partition Functions and Thermodynamic Properties of High-Temperature
Mars-Atmosphere Species from $50 \mathrm{~K}$ to $50000 \mathrm{~K}$, ESA STR-246 (ESA Publications Division, ESTEC, Noordwijk, The Netherlands, 2005).

${ }^{36}$ E. Kustova, A. Aliat, and A. Chikhaoui, Chem. Phys. Lett. 344, 638 (2001).

${ }^{37}$ A. Aliat, A. Chikhaoui, and E. Kustova, Phys. Rev. E 68, 056306 (2003).

${ }^{38}$ G. Colonna, L. D. Pietanza, and G. dAmmando, Chem. Phys. 398, 37 (2012).

${ }^{39}$ J. Ferziger and H. Kaper, Mathematical Theory of Transport Processes in Gases (North-Holland, Amsterdam, London, 1972).

${ }^{40}$ E. Kustova and D. Giordano, Chem. Phys. 379, 83 (2011).

${ }^{41}$ E. Kustova and G. Oblapenko, Phys. Fluids 27, 016102 (2015).

${ }^{42}$ E. Kustova and G. Oblapenko, Phys. Rev. E 93, 033127 (2016).

${ }^{43}$ E. Kustova, E. Nagnibeda, T. Alexandrova, and A. Chikhaoui, Chem. Phys. 276, 139 (2002).

${ }^{44}$ I. Armenise and E. Kustova, Chem. Phys. 428, 90 (2014).

${ }^{45}$ O. Kunova, E. Kustova, M. Mekhonoshina, and E. Nagnibeda, Chem. Phys. 463, 70 (2015).

${ }^{46} \mathrm{~A}$. Ern and V. Giovangigli, Multicomponent Transport Algorithms, Lecturer Notes Physics, Series Monographs, M24 (Springer-Verlag, 1994).

${ }^{47}$ E. Mason and L. Monchick, J. Chem. Phys. 36, 1622 (1962).

${ }^{48}$ D. Bruno, M. Capitelli, C. Catalfamo, R. Celiberto, G. Colonna, P. Diomede, D. Giordano, C. Gorse, A. Laricchiuta, S. Longo, D. Pagano, and F. Pirani, Transport Properties of High-Temperature MarsAtmosphere Components, ESA STR 256 (ESA, ESA Publications Division, Noordwijk, 2008).

${ }^{49}$ J. Hirschfelder, J. Chem. Phys. 26, 282 (1957).

${ }^{50}$ J. Kunc and F. Gordillo-Vazquez, J. Phys. Chem. 101, 1595 (1997).

${ }^{51}$ O. Kunova and E. Nagnibeda, Chem. Phys. Lett. 625, 121 (2015).

${ }^{52}$ A. Cenian, A. Chernukho, and V. Borodin, Contrib. Plasma Phys. 35, 273 (1995).

${ }^{53}$ M. Capitelli, C. Ferreira, B. Gordiets, and A. Osipov, Plasma Kinetics in Atmospheric Gases, Springer Series on Atomic, Optical and Plasma Physics, Vol. 31 (Springer-Verlag, Berlin, 2000).

${ }^{54}$ W. Hermann and E. Schade, Z. Phys. 233, 333 (1970).

${ }^{55}$ U. Plantikow, Z. Phys. 237, 388 (1970).

${ }^{56}$ J. Morris, R. Rubis, and J. Yos, Phys. Fluids 13, 608 (1970).

${ }^{57}$ R. Brokaw, J. Chem. Phys. 32, 1005 (1960).

${ }^{58}$ J. Butler and R. Brokaw, J. Chem. Phys. 26, 1636 (1957).

${ }^{59}$ G. G. Stokes, Trans. Cambridge Philos. Soc. 8, 287 (1845).

${ }^{60}$ L. Mandelshtam and M. Leontovich, Russ. J. Exp. Theor. Phys. 7, 438 (1937).

${ }^{61}$ L. Tisza, Phys. Rev. 61, 531 (1942).

${ }^{62}$ V. Istomin, E. Kustova, and M. Mekhonoshina, "Rarefied gas dynamics," AIP Conf. Proc. 1628, 1229-1236 (2014).

${ }^{63}$ D. Olynick, W. Henline, L. Chambers, and G. Candler, AIAA Paper No. 94-1955, 1994.

${ }^{64} \mathrm{R}$. Mc Kenzie and J. Arnold, AIAA Paper No. 67-322, 1967.

${ }^{65}$ S. Selle and U. Riedel, AIAA Paper No. 2000-0211, 2000.

${ }^{66}$ G. Chernyi, S. Losev, S. Macheret, and B. Potapkin, Physical and Chemical Processes in Gas Dynamics, Vol. 1, 2 (American Institute of Aeronautics and Astronautics, 2004).

${ }^{67}$ V. E. Gollant, A. P. Zhilnskiy, and S. A. Sakharov, Fundamentals of Plasma Physics (Atomizdat, Moscow, 1977) (in Russian).

${ }^{68}$ J. D. Ramshaw and C. H. Chang, Plasma Chem. Plasma Process. 13, 489 (1993).

${ }^{69}$ C. Park, J. Howe, R. Howe, R. Jaffe, and G. Candler, J. Thermophys. Heat Transfer 8, 9 (1994).

${ }^{70}$ T. J. Scanlon, C. White, M. K. Borg, R. C. Palharini, E. Farbar, I. D. Boyd, J. M. Reese, and R. E. Brown, AIAA J. 53, 1670 (2015). 